\title{
Saturated phosphatidic acids mediate saturated fatty acid-induced vascular calcification and lipotoxicity
}

\author{
Masashi Masuda, ${ }^{1}$ Shinobu Miyazaki-Anzai, ${ }^{1}$ Audrey L. Keenan, ${ }^{1}$ Kayo Okamura, ${ }^{1}$ Jessica Kendrick, ${ }^{1}$ Michel Chonchol, ${ }^{1}$ \\ Stefan Offermanns, ${ }^{2}$ James M. Ntambi, ${ }^{3}$ Makoto Kuro-0, ${ }^{4,5}$ and Makoto Miyazaki ${ }^{1,6}$ \\ 'Division of Renal Diseases and Hypertension, University of Colorado Denver, Aurora, Colorado, USA. ${ }^{2}$ Max Planck Institute for Heart and Lung Research, Department of Pharmacology, Bad Nauheim, \\ Germany. ${ }^{3}$ Department of Biochemistry and Nutritional Sciences, University of Wisconsin-Madison, Madison, Wisconsin, USA. ${ }^{4}$ Department of Pathology, and Charles and Jane Pak Center for Mineral \\ Metabolism and Clinical Research, University of Texas Southwestern Medical Center, Dallas, Texas, USA. ${ }^{5}$ Center for Molecular Medicine, Jichi Medical University, Tochigi, Japan. ${ }^{6}$ Division of Endocrinology, \\ Diabetes and Metabolism, University of Colorado Denver, Aurora, Colorado, USA
}

\begin{abstract}
Recent evidence indicates that saturated fatty acid-induced (SFA-induced) lipotoxicity contributes to the pathogenesis of cardiovascular and metabolic diseases; however, the molecular mechanisms that underlie SFA-induced lipotoxicity remain unclear. Here, we have shown that repression of stearoyl-CoA desaturase (SCD) enzymes, which regulate the intracellular balance of SFAs and unsaturated FAs, and the subsequent accumulation of SFAs in vascular smooth muscle cells (VSMCs), are characteristic events in the development of vascular calcification. We evaluated whether SMC-specific inhibition of SCD and the resulting SFA accumulation plays a causative role in the pathogenesis of vascular calcification and generated mice with SMC-specific deletion of both Scd1 and Scd2. Mice lacking both SCD1 and SCD2 in SMCs displayed severe vascular calcification with increased ER stress. Moreover, we employed shRNA library screening and radiolabeling approaches, as well as in vitro and in vivo lipidomic analysis, and determined that fully saturated phosphatidic acids such as 1,2-distearoyl-PA (18:0/18:0-PA) mediate SFA-induced lipotoxicity and vascular calcification. Together, these results identify a key lipogenic pathway in SMCs that mediates vascular calcification.
\end{abstract}

\section{Introduction}

Vascular calcification is a major complication in patients who are aging, have diabetes, or have chronic kidney disease (CKD) and is an active process that differentiates vascular smooth muscle cells (VSMCs) into osteoblast-like cells $(1,2)$. This process is highly regulated by transcription factors involved in osteogenic differentiation, such as RUNX2, MSX2, and ATF4 (3-5). Several in vitro and in vivo studies have shown lipids to play a causative role in the pathogenesis of vascular calcification in addition to inorganic phosphate, inflammatory cytokines, and oxidative stress (6-13). Treatment with unsaturated fatty acids (UFAs) inhibits vascular mineralization and osteogenic differentiation $(14,15)$, whereas oxidized lipids, such as oxysterols and oxidized phospholipids, elicit procalcific effects in vascular cells $(9,16,17)$. In addition to this evidence, we also previously reported that saturated fatty acids (SFAs) and calcium simultaneously accumulate during osteogenic differentiation of vascular cells $(18,19)$.

Ectopic accumulation of excess lipids, called lipotoxicity, plays a central role in the pathogenesis of cardio-metabolic diseases, including diabetes, obesity, atherosclerosis, and vascular calcification (20-24). However, evidence from a number of experimental systems is emerging, stating that SFAs and UFAs have distinct contributions to lipotoxicity $(25,26)$. SFAs such as palmitic acid (16:0) and stearic acid (18:0) induce apoptosis, oxidative stress, and ER stress in a variety of mammalian cell lines (including hepatocytes,

Conflict of interest: The authors have declared that no conflict of interest exists. Submitted: May 18, 2015; Accepted: September 10, 2015.

Reference information: J Clin Invest. 2015;125(12):4544-4558. doi:10.1172/JCI82871. macrophages, and VSMCs), whereas UFAs such as oleic acid have no or minimal lipotoxic properties $(5,25-30)$. In addition, cotreatment with UFAs blocks SFA-mediated lipotoxic effects $(25,29)$. However, the specific metabolite of SFAs that induces lipotoxicity, the mechanism underlying SFA-mediated lipotoxicity, and how UFAs block SFA-mediated lipotoxicity are largely unknown.

Proper intracellular SFA and UFA balance is controlled by a lipogenic enzyme called stearoyl-CoA desaturase (SCD) (31, 32). SCD catalyzes the conversion of SFAs to monounsaturated FAs, mainly 16:0 into palmitoleate (16:1n-7), and 18:0 into oleate $(18: 1 n-9)(33,34)$. This introduction of a double bond markedly impacts several chemical properties, including a decrease in melting point and an increase in solubility. The activation of SCD therefore neutralizes SFA-mediated lipotoxicity $(5,25)$. The expression of SCD is highly regulated by a number of hormonal and dietary factors $(33,35-37)$. Recently, we found that the accumulation of SFAs by either supplementation with exogenous SFAs or inhibition of SCD induces mineralization of VSMCs $(5,19)$. In addition, SFA-mediated lipotoxicity and vascular calcification were completely blocked by an acyl-CoA synthetase inhibitor and were attenuated by the shRNA-mediated inhibition of fatty acid elongase-6 (Elovl6) (5), suggesting that stearoyl-CoA (18:0-CoA) or its metabolite contributes to mineralization of VSMCs. The majority of 18:0-CoA is utilized as substrate for acyltransferases, such as glycero-3-phosphate acyltransferase (GPAT) and 1-acylglycerol acyltransferase (AGPAT), and incorporated into phospholipid and neutral lipid fractions. However, which metabolite of 18:0-CoA mediates the lipotoxic and procalcific effects has not been identified. 
In this study, we examined whether (i) accumulation of SFAs in VSMCs through SCD reduction is a common feature in vascular calcification and (ii) whether the inhibition of SCD specifically in SMCs causes vascular calcification in vivo. Finally, we identified SFA-derived metabolites that are responsible for lipotoxicity and vascular calcification.

\section{Results}

Reduced SCD activity is common in VSMCs of murine models of vascular calcification. The reduction of klotho, an aging-related protein, is considered to be a major reason that patients with CKD have a markedly higher prevalence of vascular calcification (38, 39). We previously reported that accumulation of SFAs, particularly 18:0, through SCD inhibition induced vascular calcification in vitro (5). To determine whether the inhibition of SCD induced by klotho deficiency is a major event in vascular calcification, we used immunomagnetic cell sorting to isolate VSMCs from klotho-deficient (Klotho ${ }^{-/}$) mice with the SMMHC-GFP gene (SMMHC-GFP; $\mathrm{Klotho}^{-/}$) and WT mice (SMMHC-GFP; $\left.\mathrm{Klotho}^{+/+}\right)$. As expected, Klotho ${ }^{-/-}$mice developed severe hyperphosphatemia, high fibroblast growth factor 23 (FGF23) circulating levels, and aortic medial calcification (Supplemental Figure 1, A-C; supplemental material available online with this article; doi:10.1172/JCI82871DS1). Quantitative PCR (qPCR) analysis showed that VSMCs highly expressed $S c d 1$ and $S c d 2$ isoforms, but both of these isoforms were significantly reduced in $\mathrm{Klotho}^{-1}$ mice compared with WT controls (Figure 1A). SCD1 and SCD2 protein levels were also diminished in $\mathrm{Klotho}^{-/-}$mice (Figure 1B). Consistently, $\Delta 9$ desaturase activity was significantly reduced in $\mathrm{Klotho}^{-/-}$mice (Figure 1C). In addition, desaturation index (16:1/16:0 and 18:1/18:0) levels decreased in the aortic medial layers of $\mathrm{Klotho}^{-/-}$mice (Figure 1D). Since excess calcitriol and inorganic phosphate are major contributors to vascular calcification induced by klotho deficiency $(40,41)$, we examined whether calcitriol, phosphate, and their combination affects SCD expression in human VSMCs. Two SCD isoforms (SCD and SCD5) are present in the human genome (42). Human VSMCs express SCD but not SCD5 protein (data not shown). Treatment of VSMCs with high phosphate $(2.0 \mathrm{mM})$ and calcitriol reduced levels of $S C D$ mRNA by $59 \%$ and $33 \%$, respectively (Figure $1 \mathrm{E}$ ). Moreover, a combination of high phosphate plus calcitriol reduced SCD mRNA, activity, and protein by over $80 \%$ (Figure 1, E-H).

We also examined whether aortic SCD activity and expression are reduced in another murine model of vascular calcification, DBA/2J mice with CKD (12). DBA/2J mice with 5/6 nephrectomies developed increased levels of serum creatinine, phosphorus, FGF23, and severe medial calcification consistent with a CKD phenotype. CKD DBA/2J mice had significantly lower aortic $S c d 1$ and $S c d 2$ expression compared with DBA2J with sham operations (normal kidney function, Supplemental Figure 2, A-G). CKD reduced the activity of SCD by $88 \%$ (Supplemental Figure $2 \mathrm{H}$ ). We previously reported that a $50 \%$ reduction of SCD activity is sufficient to induce mineralization of VSMCs (5). Thus, we concluded that aortic 18:0 accumulation by a reduction of SCD1 and SCD2 isoforms is a significant event in the development of vascular calcification. In support of the results from the animal studies, human subjects with stage 3 and 4 CKD had higher levels of serum
18:0 but not oleic acid, as well as a consequent lower desaturation index that is reflective of SCD activity compared with subjects with normal kidney function, suggesting that CKD reduces SCD activity in humans (Supplemental Figure 2, I-K).

Dual inhibition of SCD1 and SCD2 induces mineralization, osteogenic differentiation, and ER stress of VSMCs in vitro and in vivo. We previously reported that treatment of VSMCs with an SCD pan inhibitor (SCDi), CAY10566, induced mineralization and osteogenic differentiation. However, which SCD isoform contributes to vascular calcification has not been determined. To determine which SCD isoform is more important in mediating vascular calcification, SCD1 and SCD2 isoforms were inhibited in VSMCs using shRNAs. Simultaneous inhibition of $S c d 1$ and $S c d 2$, but not $S c d 1$ or $S c d 2$ single inhibition, induced mineralization and alkaline phosphatase (ALP) activity (a marker for osteogenic differentiation) of VSMCs. In this study, we used the mouse VSMC, MOVAS-1, line because other VSMC lines - such as human primary VSMCs, human VSMC SM2, mouse and rat primary VSMCs, and bovine calcifying vascular cells - were either not resistant to or morphologically altered in the presence of lentiviral shRNA and puromycin selection.

$S c d 1$ and $S c d 2$ shRNA efficiently reduced levels of $S c d 1$ and $S c d 2$ mRNA (Supplemental Figure 3A). Interestingly, simultaneous inhibition of $S c d 1$ and $S c d 2$ was required for inducing mineralization and osteogenic differentiation of VSMCs (Supplemental Figure 3, B-D). We previously reported that 18:0-induced ER stress contributed to vascular calcification (5). $S c d 1 / 2$ dual inhibition, but not $S c d 1$ or $S c d 2$ single inhibition, drastically induced levels of phospho-protein kinase R-like endoplasmic reticulum kinase (p-PERK), CCAAT-enhancer-binding protein homologous protein (CHOP), spliced X-box binding protein (sXBP-1), and activating transcription factor-4 (ATF4) protein and mRNA, indicating that $S c d 1 / 2$ dual inhibition is required for causing ER stress (Supplemental Figure 3, E and F). We also found that $S c d 1 / 2$ dual inhibition induced the production of inflammatory cytokines in VSMCs. These data suggest that dual inhibition of $S c d 1$ and $S c d 2$, rather than the inhibition of a single $S c d$ isoform, contributes to mineralization of VSMCs, as well as the activation of ER stress.

To examine whether ER stress directly contributes to vascular calcification, human VSMCs were treated with 2 chemicals that specifically induce ER stress, tunicamycin (a specific inhibitor of N-linked glycosylation) and thapsigargin (a noncompetitive inhibitor of the sarco/ER Ca ${ }^{2+}$ ATPase). Both ER stress inducers significantly induced mineralization of VSMCs (Supplemental Figure $3 G$ ). We also examined which of the 3 ER stress pathways contributes to SCD inhibition-induced mineralization. As shown in Supplemental Figure 3, H-J, Atf6, Ire1, and Perk shRNAs reduced the expression of their target genes by $52 \%, 81 \%$, and $68 \%$, respectively. In addition to Atf4 and Chop knockdown (5), Atf6, Ire1, and Perk knockdown attenuated SCD inhibitioninduced mineralization (Supplemental Figure 3, K-M). In addition, Atf6 and Perk knockdowns reduced mineralization even in the absence of SCDi (Supplemental Figure 3, K-M). These results suggest that all $3 \mathrm{ER}$ stress response pathways synergistically induce vascular calcification in response to SCD inhibition.

Since our in vitro studies indicated that dual inhibition of $S c d 1$ and $S c d 2$ was required for inducing vascular calcification, 

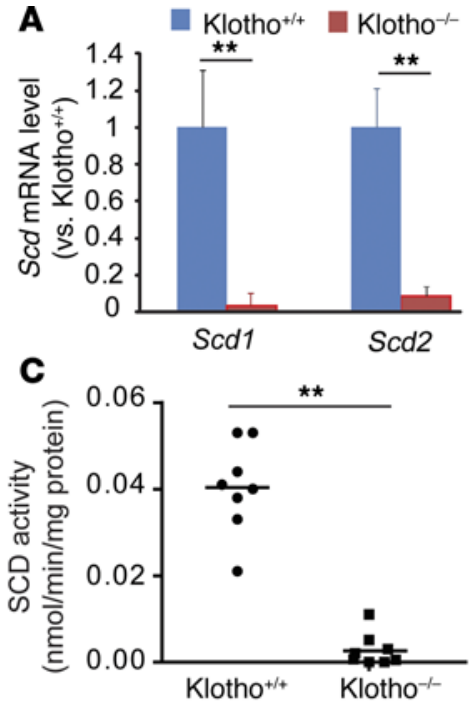

E

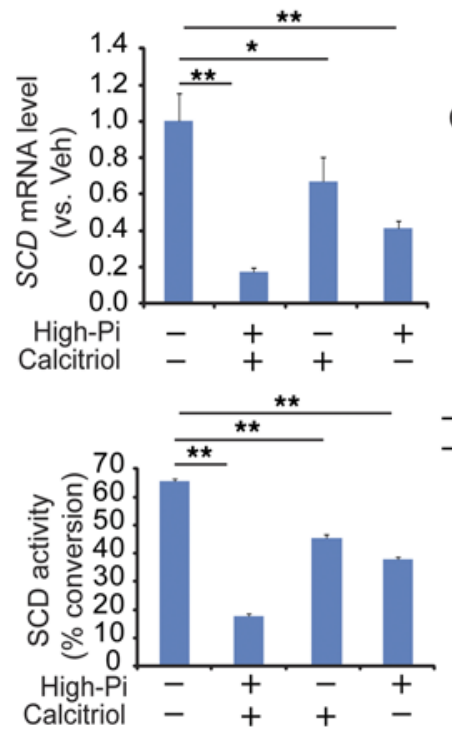

B

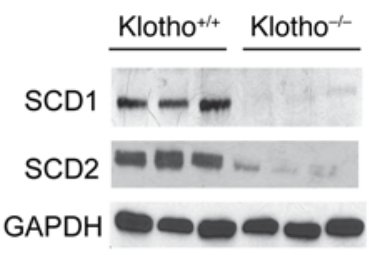

D $\mathrm{Klotho}^{+/+} \quad \mathrm{Klotho}^{--}$

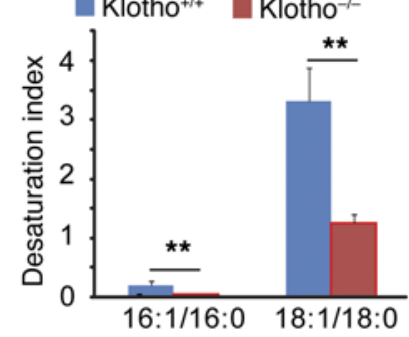

$\mathbf{F}$
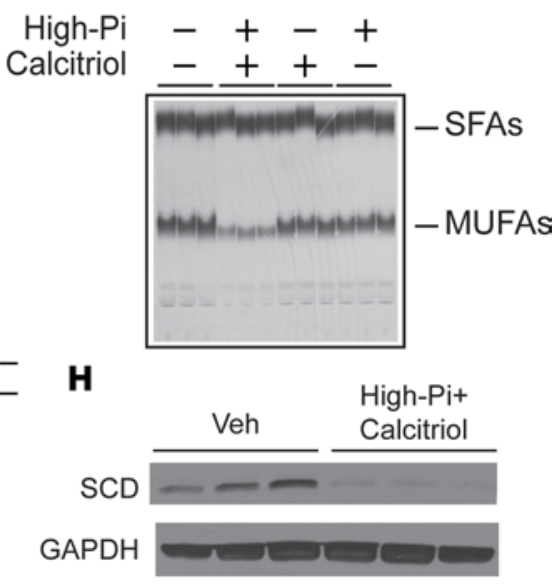

Figure 1. Klotho deficiency reduces the expression of SCD1 and SCD2 in VSMCs. Eight-week-old male SMMHC-GFP; $\mathrm{Klotho}^{-/-}\left(\mathrm{Klotho}^{--}\right)$mice and SMMHC-GFP; $\mathrm{Klotho}^{+/+}\left(\mathrm{Klotho}^{+/+}\right)$mice $(n=8)$ were sacrificed after a 4-hour fasting. (A) Levels of Scd1 and Scd2 mRNA in the aortas of SMMHC-GFP; $\mathrm{Klotho}^{-1-}$ mice and SMMHC-GFP; $\mathrm{Klotho}^{+/+}$mice. VSMCs were isolated as GFP+ cells by immunomagnetic cell sorting. Scd1 and Scd2 RNA expression was determined by QPCR. (B) Immunoblot analysis of SCD1 and SCD2 protein. Total protein extract was prepared from the aortas and subjected to immunoblot analysis with SCD1- and SCD2-sepcific antibodies. (C) Microsomal SCD activity in the aortas. Microsomal protein $(100 \mu \mathrm{g})$ was incubated with ${ }^{14} \mathrm{C}-18: 0$-CoA in the presence of NADH. SCD activity was determined as the conversion of 18:0-CoA to 18:1n-9. (D) Desaturation index in the medial layer of aortas from SMMHC-CFP; Klotho $^{-1-}$ mice and SMMHC-CFP; Klotho ${ }^{+/+}$mice. Levels of fatty acids were determined by gas chromatography analysis. (E) High-phosphate and calcitriol reduced SCD mRNA in human VSMCs. (F) Autoradiography and (G) quantification of SCD activity in VSMCs treated with high-phosphate $(\mathrm{Pi})$ and calcitriol. (H) Combination of high-phosphate and calcitriol reduced levels of SCD protein. Human VSMCs were treated with $2.0 \mathrm{mM}$ phosphate and 100 nM calcitriol for 7 days. ${ }^{* *} P<0.001$ vs. Klotho ${ }^{+/+}$mice (2-tailed Student's $t$ test).

SMC-specific $S c d 1 / 2 \mathrm{KO}$ mice displayed severe vascular calcification in both aortic sinus and arch lesions, whereas vascular calcification was not observed in the control mice (Figure 3, A-D). Atherosclerotic lesions were not found in either the control or SMC-Scd1/2 KO mice (data not shown). Consistent with histological analysis, aortic calcium content was significantly (>4.8-fold) higher in SMC-Scd1/2 KO mice (Figure 3D). In support of these data, we found that SMC-Scd1/2 deficiency increased mRNA levels of osteogenic makers such

we next determined the role of SCD1 and SCD2 dual inhibition in the pathogenesis of vascular calcification in vivo. Since global $S c d 1$ and $S c d 2$ double KO mice are embryonic lethal, we generated SMC-specific $S c d 1$ and $S c d 2$ dual inducible KO (SMC-Scd1/2 KO) mice by crossing $S c d 1 / 2$ dual floxed mice with $S M M H C-C r e^{E R(T 2)}$ mice. Only males were used for the studies because the SMMHC-Cre ${ }^{E R(T 2)}$ transgene was inserted onto the Y chromosome. The SMC-specific Scd1/2 dual conditional mice were i.p. injected with tamoxifen or vehicle to generate SMC-specific $S c d 1 / 2 \mathrm{KO}$ mice and control mice, respectively. Mice injected with tamoxifen showed over a $99 \%$ reduction of $S c d 1$ and $S c d 2$ mRNA and protein levels in the medial layer of aortas (Figure 2, A and B), resulting in a drastic reduction of SCD activity (Figure 2C). Levels of mono-UFAs (MUFAs) such as 16:1n-7 and 18:1n-9 were reduced in the medial layer of aortas of SMC-Scd1/2 KO mice, whereas SFA levels such as 16:0 and 18:0 were significantly increased (Figure 2D). Levels of hepatic $S c d 1$ and $S c d 2$ mRNA were comparable between SMC-Scd1/2 KO and control mice. There were no differences in body weight, serum cholesterol, serum triacylglycerol (TAG), serum calcium, serum creatinine, and serum phosphorus between SMC-Scd1/2 KO mice and control mice (data not shown). as $A l p$, osteocalcin (Ocn), osteopontin (Opn), and type III phosphate transporter-1 (PiT1), and reduced SMC markers such as $S m 22$ and Smmhc (Figure 3E). Along with the induction of vascular calcification, SMC-specific $S c d$ deficiency also induced ER stress in the VSMCs of SMC-Scd1/2KO mice (Figure 3, E-G). qPCR and immunoblot analysis revealed that mRNA and protein levels of ER stress markers such as Atf4, Chop, sXbp-1, Gadd34, and Atf3 were significantly increased in the VSMCs of SMC-Scd1/2 KO mice (Figure 3, E and F). We previously demonstrated that CHOPinduced cell apoptosis contributes to the pathogenesis of vascular calcification (9). Immunofluorescence analysis showed that apoptotic cell death and CHOP expression is significantly higher in the aortic sinuses of SMC-Scd1/2 KO mice compared with control mice (Figure 3, G-I).

GPAT4, AGPAT3, and AGPAT5 contribute to SFA-mediated vascular calcification and lipotoxicity. Since both in vitro and in vivo studies indicated that SCD inhibition strikingly induced ER stress and vascular calcification, we next strived to elucidate the molecular mechanism by which SCD inhibition induces ER stress-mediated vascular calcification in VSMCs. Previously, we reported that the accumulation of SFAs, especially 18:0 through the inhibition 
A

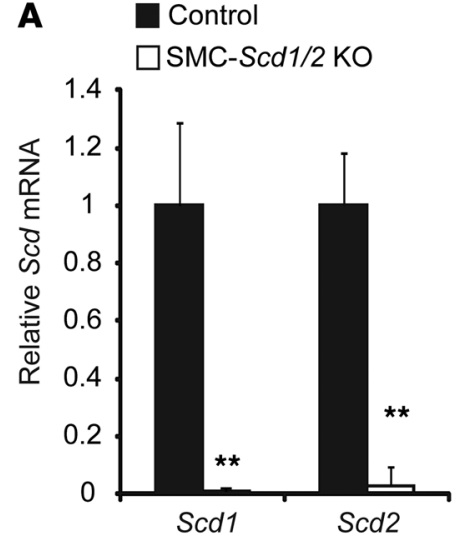

C

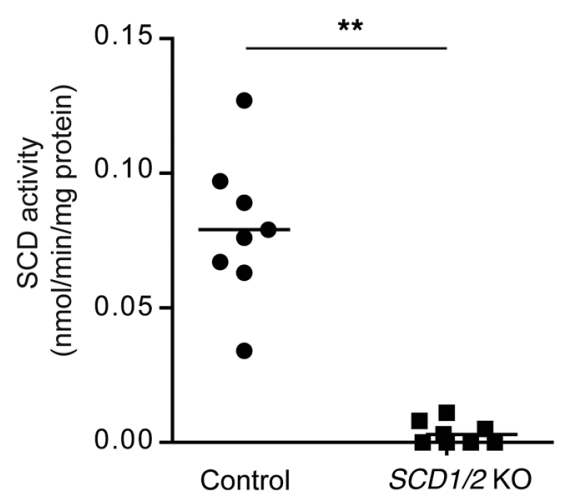

B

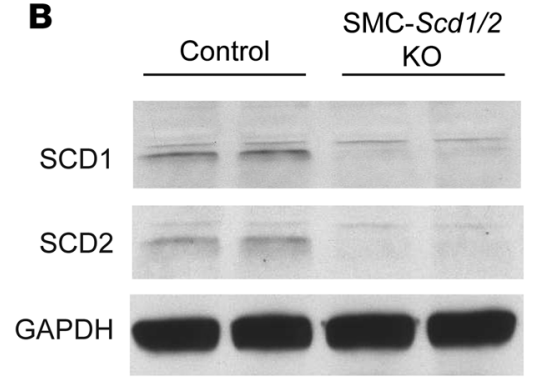

Figure 2. Generation of SMC-Scd1/2 KO mice. (A) Levels of $S c d 1$ and $S c d 2$ mRNA in the medial layer of aortas of SMC-Scd1/2 KO mice and control mice $(n=8)$. Scd1 and Scd2 RNA expression was determined by qPCR. (B) Immunoblot analysis of SCD1 and SCD2 proteins. Total protein extracts were prepared from the medial layer of the aortas and subjected to immunoblot analysis with SCD1- and SCD2-specific antibodies. (C) Microsomal SCD activity in the medial layer of aortas. Microsomal protein $(100 \mu \mathrm{g})$ was incubated with ${ }^{14} \mathrm{C}-18: 0$-CoA in the presence of NADH. SCD activity was determined as the conversion of 18:0-CoA to 18:1n-9. (D) Fatty acid content in the medial layer of aortas from SMC-Scd1/2 KO mice and control mice. ${ }^{* *} P<0.001$ vs. control mice (2-tailed Student's $t$ test)

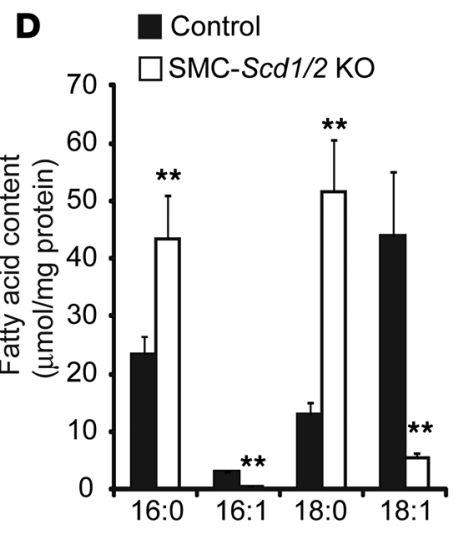

Transient knockdowns of acyltransferases in human cells using siRNA and shRNA were also performed. However, the efficiency of knockdown was more effective and consistent using stable cell lines than transient knockdowns. Since VSMCs are difficult to transfect with plasmids and oligos, the knockdown efficiency was only $17 \%-55 \%$ and there were considerable variations in the knockdown efficiencies between genes. To induce vascular calcification and ER stress, we treated all acyltransferase knockdown VSMCs with $200 \mu \mathrm{M}$ 18:0. Treatment with 18:0 significantly induced mineralization in control VSMCs containing empty shRNA, whereas Gpat4, Agpat3,

of SCD, results in ER stress and vascular calcification (5). In addition, our previous studies indicated that SCD inhibition and 18:0mediated ER stress and vascular calcification were completely abolished in the presence of Triacsin C, a potent pan inhibitor of acyl-CoA synthetase (5). 18:0 must be converted to 18:0-CoA to elicit lipotoxic effects. Since over 70\% of generated 18:0-CoA from VSMCs treated with SCDi and Scd1/2 shRNA is incorporated to acyl-lipid fractions as a substrate for acyltransferases, we hypothesized that an acyl-lipid containing 18:0 mediates SFA-induced ER stress and vascular calcification. To identify a 18:0 metabolite responsible for lipotoxicity and vascular calcification, we used 3 approaches: (i) screening an acyltransferase shRNA library to identify which acyltransferase contributes to 18:0-mediated vascular calcification and ER stress, (ii) determining ${ }^{14} \mathrm{C}-18$ :0 lipid partitioning in VSMCs to examine which lipid species mainly incorporates 18:0, and (iii) identifying which lipid species increases with SCD inhibition by in vitro and in vivo liquid chromatography-mass spectrometry-based (LC-MS-based) lipidomics.

To identify acyltransferases that are responsible for 18:0mediated vascular mineralization and lipotoxicity, we first analyzed levels of mRNA expression of mammalian acyltransferases using qPCR analysis with the absolute standard curve method. As shown in Supplemental Table 1, 21 of 27 lipid acyltransferases that are present in the mammalian genome were expressed in VSMCs. To screen all of the acyltransferases expressed in VSMCs, we inhibited each acyltransferase using a customized acyltransferase shRNA knockdown library. All shRNAs efficiently reduced the expression of their target acyltransferases by over 75\% (Supplemental Figure 4). and Agpat 5 knockdowns were the only acyltransferase knockdowns to significantly reduce 18:0-mediated vascular calcification (Figure 4A). In addition, Gpat4, Agpat3, and Agpat5 knockdowns significantly reduced 18:0-induced Chop expression (Figure 4B). We also used several inhibitors or activators such as ACAT (Sandoz 58-035), ceramide synthase (Fumonisin B1), fatty acid oxidation (etomoxir), and a DGAT1 inhibitor (A-92250) to block 18:0 metabolism. Although Triascin C completely inhibited 18:0-induced mineralization, none of these inhibitors blocked 18:0-mediated vascular calcification (data not shown).

We further examined the contribution of GPAT4, AGPAT3, and AGPAT5 in SCD inhibition-mediated vascular calcification and lipotoxicity. While Gpat4 and Gpat1 knockdowns comparably reduced GPAT activity in VSMCs (Supplemental Figure 5, A and B), Gpat4 but not Gpat1 knockdown attenuated mineralization of VSMC in both the presence and absence of 18:0 and SCDi (Supplemental Figure 5, C-E). Since Gpat4 knockdown reduced Ocn and Opn expression, 18:0-mediated induction of osteogenic differentiation was reduced in Gpat4 but not Gpat1 knockdown cells (Supplemental Figure 5, F and G). In addition, Gpat4 knockdown reduced the expression of PiT1, which contributes to vascular calcification, resulting in the reduction of phosphate uptake (Supplemental Figure 5, H and I). SCD inhibition- and 18:0-mediated ER stress was attenuated in Gpat4 but not Gpat1 knockdown cells (Supplemental Figure 5, and CHOP protein and mRNA in control cells, whereas Gpat4 knockdown significantly attenuated the induction of ATF4, J-N). Treatment with 18:0 and SCDi increased levels of ATF4 

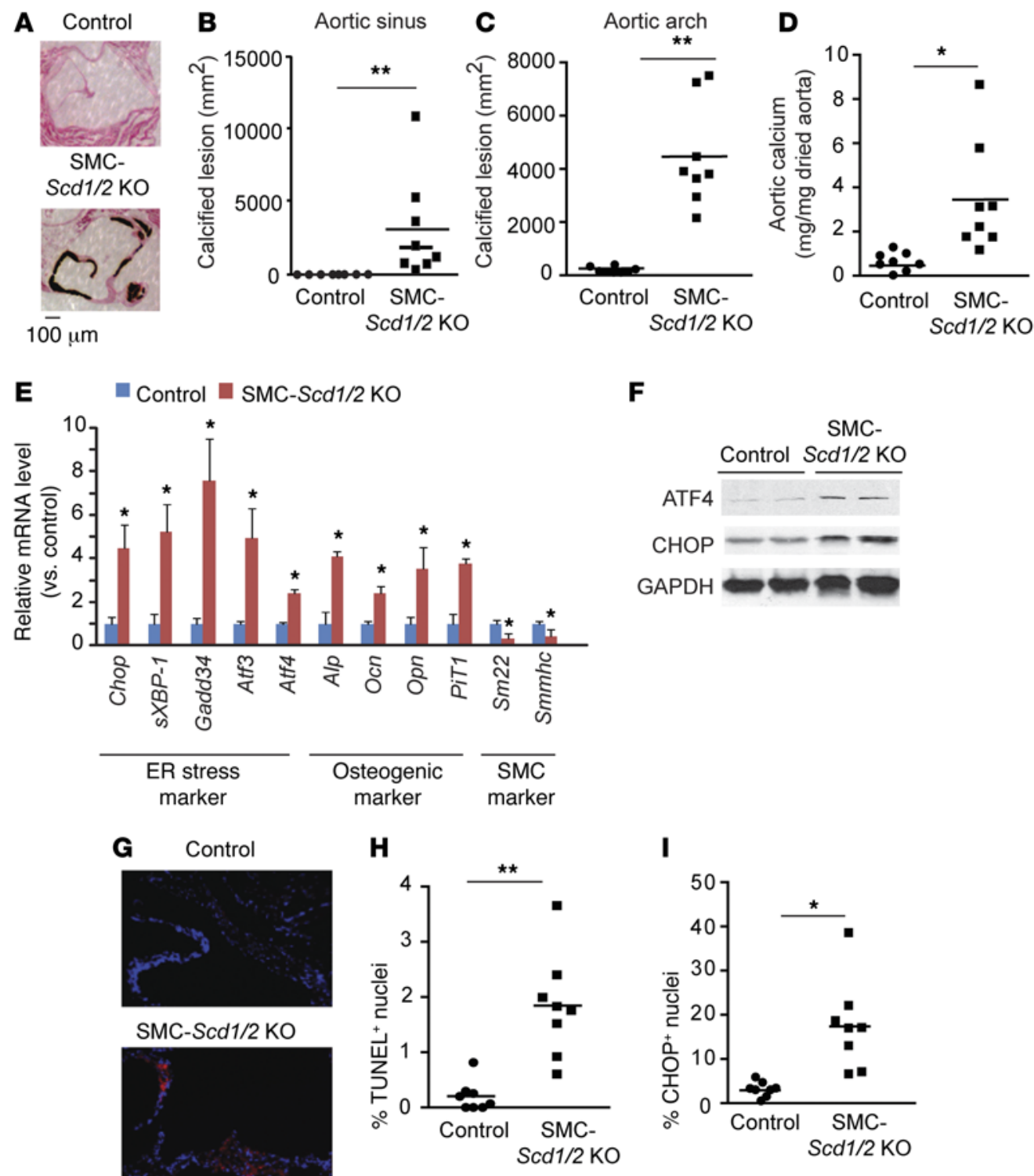

$\overline{100 \mu \mathrm{m}}$
I

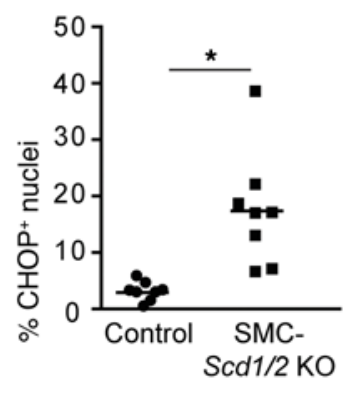

Figure 3. SMC-specific SCD1 and SCD2 double deficiency induces vascular calcification, ER stress, and vascular apoptosis. (A) Representative photograph $(\times 10)$ of the lesions of aortic sinuses stained with von Kossa. Mice $(n=8)$ were sacrificed after 10 weeks of tamoxifen injections. Quantitative analysis of calcified lesions in the (B) aortic sinus and (C) aortic arch. (D) Aortic calcium content in SMC-Scd1/2 KO mice. (E) mRNA levels of ER stress, osteogenic differentiation, and SMC markers in VSMCs. VSMCs were isolated by immunomagnetic cell sorting. Scd1 and Scd2 mRNA expression was determined by qPCR. (F) Immunoblot analysis of ATF4 and CHOP protein expression in the medial layer of aortas of SMC-Scd1/2 KO mice. (G) Representative micrographs show more TUNEL ${ }^{+}$signal (red) in nuclei (blue) of aortic sinus lesions from control and SMC-Scd1/2 KO mice. (H) Quantitative analysis of TUNEL ${ }^{+}$nuclei conducted on lesions from SMC-Scd1/2 KO mice. (I) Immunofluorescence analysis of CHOP in the aortic sinuses of SMC-Scd1/2 KO mice. ${ }^{*} P<0.01$ and ${ }^{* *} P<0.001$ vs. control mice (2-tailed Student's $t$ test).
CHOP, and sXBP-1 expression in response to 18:0 and SCDi treatment (Supplemental Figure 5, J-N).

To confirm the contribution of GPAT4 in SFA-induced vascular calcification and lipotoxicity, we overexpressed human GPAT4 in VMSCs using a tetracylcine-controlled transcriptional activation (Tet-on) system. Doxycycline treatment induced GPAT4 expression, resulting in an increase in total GPAT activity by approximately 2.5-fold (Supplemental Figure 6, A and B). In contrast to Gpat4 knockdown, GPAT4 overexpression induced mineralization (Supplemental Figure 6C) and osteogenic differentiation (Supplemental Figure 6, D and E) in the absence and presence of 18:0 and SCDi. In addition, GPAT4 overexpression increased phosphate uptake (Supplemental Figure 6F) and ER stress in response to SCDi treatment (Supplemental Figure 6, G-I).

Similar to Gpat4 knockdown, Agpat3 and Agpat5 knockdowns inhibited SCDi-mediated mineralization of VSMCs (Supplemental Figure 7, A and B). Levels of osteogenic markers such as Alp and Ocn, as well as PiT1, were reduced in Agpat3 and Agpat5 knockdown cells treated with SCDi (Supplemental Figure 7, C-H). In addition, Agpat3 knockdown completely reduced SCD inhibition-mediated induction of ATF4 and CHOP proteins (Supplemental Figure 7I). Levels of ATF4 and CHOP proteins were also substantially lower in Agpat5 knockdown cells treated with SCDi, although Agpat3 knockdown was more effective than Agpat5 knockdown (Supplemental Figure 7J). GPAT4, AGPAT3, and AGPAT5 catalyze the initial 2 steps of de novo glycerolipid biosynthesis and generate phosphatidic acid (PA) in the ER (Figure 5A and ref. 43). In addition, the inhibition of acyl-CoA: cholesterol acyltransferase-1 (ACAT1) and ACAT2 responsible for cholesteryl ester (CE) synthesis, diacylglycerol acyltransferase-1 (DGAT1) and DGAT2 responsible for triacylglycerol synthesis, monoacylglycerol acyltransfease-1 (MOGAT1) responsible for diacylglycerol (DAG) synthesis, and serine palmitoyltransferase (SPTLC1) responsible for sphingolipid and ceramide synthesis 


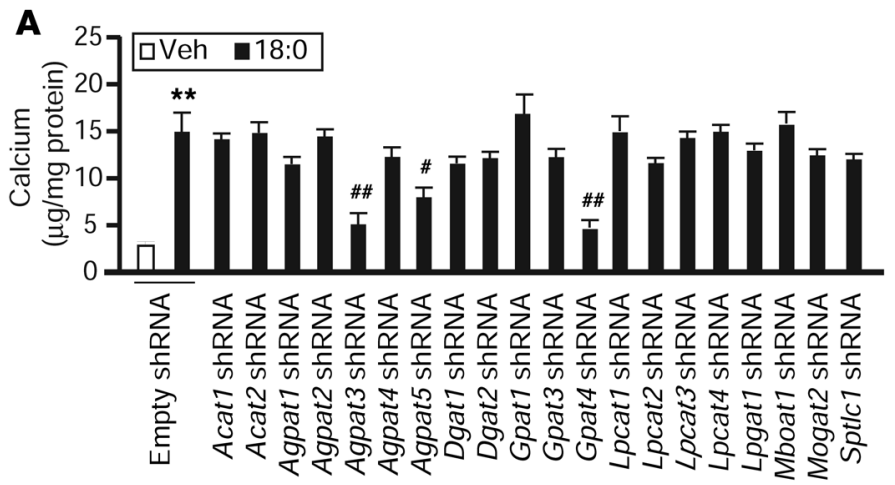

B

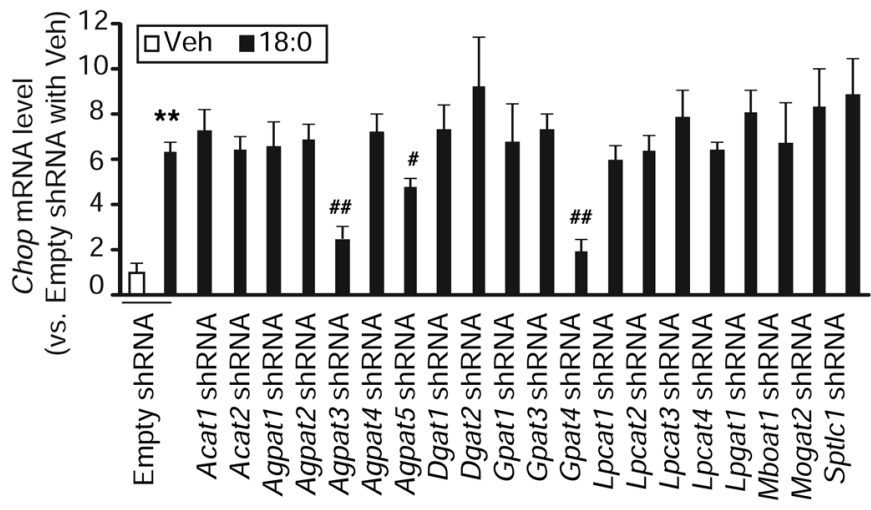

did not affect 18:0-induced vascular calcification and lipotoxicity. These results suggest that PA or its downstream metabolite, glycerophospholipid containing 18:0, contributes to 18:0mediated vascular calcification and lipotoxicity.

To determine whether PA or glycerophospholipid contributes to lipotoxicity, we next modified Lipin, a PA phosphatase (PAP) enzyme that participates in the third step of de novo glycerolipid synthesis and catalyzes the conversion of PA to DAG in the ER. Lipin 2 is a major isoform expressed in VSMCs (Supplemental Figure 8A). Treatment with Lipin2 shRNA reduced mRNA levels of Lipin 2 and PAP activity by $78 \%$ and $46 \%$, respectively (Supplemental Figure 8, B and C). The remaining activity is likely derived from Lipin1 and PAP2 enzymes. In Lipin 2 knockdown cells, the effects of 18:0 and SCD inhibition on mineralization and osteogenic differentiation were significantly enhanced compared with the control cells (Supplemental Figure 8, D-G). In addition, Lipin2 knockdown augmented ER stress (Supplemental Figure 8, H-K). These results suggest that PA contributes to SFA-induced mineralization and lipotoxicity.

SFAs such as 18:0 and 16:0 preferentially accumulate in PA. Based on the acyltransferase and Lipin shRNA screening, PA is a potential candidate for mediating SFA-induced lipotoxicity. We therefore hypothesized that 18:0 is preferentially incorporated into the PA fraction. Since control human VSMCs converted over $50 \%$ of 18:0 and 16:0 into MUFAs, and because VSMCs treated with SCDi had a complete inhibition on the conversion of SFAs to MUFAs (Figure 5 , B and C), SFA-specific lipid partitioning is determined only in VSMCs treated with SCDi. Under SCD inhibition, 18:0 and 16:0 are preferentially incorporated into the PA fraction in VSMCs (Figure
Figure 4. GPAT4, AGPAT3, and AGPAT5 contribute to SFA-induced mineralization and ER stress of VSMCs. ShRNA-mediated knockdown of acyltransferases in VSMCs. MOVAS-1 cells were infected with lentiviruses containing acyltransferase shRNAs for 48 hours and selected with $5 \mu \mathrm{g} / \mathrm{ml}$ puromycin to generate each acyltransferase stable knockdown VSMC. Levels of acyltransferase mRNAs were determined by qPCR. Each shRNA reduced its targeted acyltransferase by over $75 \%$. (A) Mineralization of VSMCs in the presence of 18:0. The stable acyltransferase knockdown VSMCs were treated with $200 \mu \mathrm{M}$ 18:0 as BSA complex for 7 days in the presence of $2 \mathrm{mM}$ inorganic phosphate. (B) Levels of Chop mRNA in acyltransferase stable knockdown VSMCs treated with $200 \mu \mathrm{M}$ 18:0. The stable acyltransferase knockdown VSMCs were treated with $200 \mu \mathrm{M}$ 18:0 as BSA complex for 24 hours. Levels of Chop mRNA were analyzed by $\mathrm{qPCR} .{ }^{* *} P<0.001$ vs. MOVAS -1 cells treated with empty shRNA and vehicle. $n=4 ;{ }^{\#} P<0.01$ and ${ }^{\#} P<0.001$ vs. MOVAS-1 cells treated with empty shRNA and $200 \mu \mathrm{M}$ 18:0 (one-way ANOVA).

5, D-F). The incorporation of 18:0 and 16:0 into the PA fraction was increased by $>15$-fold and $>9$-fold, respectively. Upon SCD inhibition, SFA distribution into lysophosphatidylcholine (LPC), phosphatidylinositol (PI), and lysoPA (LPA) also increased, whereas distribution into phosphatidiylethanolamine (PE) and sphingomyelin (SM) decreased (Figure 5, E and F). In addition, SCD inhibition did not affect the lipid distribution of 18:1n-9 (Supplemental Figure 9, A and B). Consistently, 18:0 distribution in $S c d 1 / 2$ knockdown MOVAS-1 cells was nearly identical to the distribution in VSMCs treated with SCDi (data not shown).

Fully saturated PAs such as 1,2-distearoyl PA (18:0/18:0-PA) and 1,2-dipalmitoyl PA (16:0/16:0-PA) accumulate in SCD knockdown VSMCs and the aortic medial layers of SMC-Scd1/2 KO mice. We next performed an LC-MS-based lipidomic quantitative analysis to determine which lipids are increased in human VSMCs in response to $\mathrm{SCD}$ inhibition in vitro and in vivo. According to the analysis, over 270 lipid species were detected in VSMCs (Supplemental Table 3). Similar to the results of the ${ }^{14} \mathrm{C}-18: 0$ distribution study, in vitro SCD inhibition most drastically increased absolute levels of PA by 23-fold compared with the control, followed by LPA and DAG levels (Figure 5G). SCD inhibition increased all fully saturated PAs (PA containing 2 SFAs in both the sn- 1 and sn-2 position) such as 16:0/18:0-PA and 18:0/18:0-PA, myristoylstearoyl-PA (14:0/18:0-PA), and 16:0/16:0-PA. Both 16:0/18:0-PA and 18:0/18:0-PA were most drastically increased by over 200fold in VSMCs treated with SCDi. Levels of fully unsaturated PAs such as dioleoyl (18:1/18:1) and partially unsaturated PAs such as palmitoyloleoyl (16:0/18:1) were not altered in VSMCs treated with SCDi (Figure 5H).

Similar to the in vitro results, in vivo quantitative lipidomic analysis was able to detect over 250 lipid species (Supplemental Table 4). Since cell sorted-VSMCs were insufficient for the lipidomic analysis, the lipid contents in the medial layer of aortas were compared between SMC-Scd1/2 KO and control mice. The in vivo lipidomic analysis revealed that levels of PA significantly increased in the medial layer of aortas of SMC-Scd1/2 KO mice compared with control mice (Figure 5I). However, levels of phosphatidylcholine, CE, and TAG were reduced in SMC-Scd1/2 KO mice (Figure 5I). SMC-Scd1/2 KO deficiency increased levels of fully saturated PAs such as 16:0/16:0-PA and 18:0/18:0-PA by 11 -fold and 63-fold, respectively, whereas levels of partially 
A Glycerol-3-phosphate B

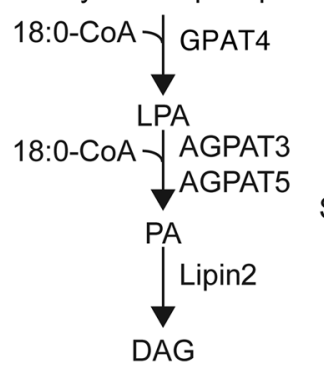

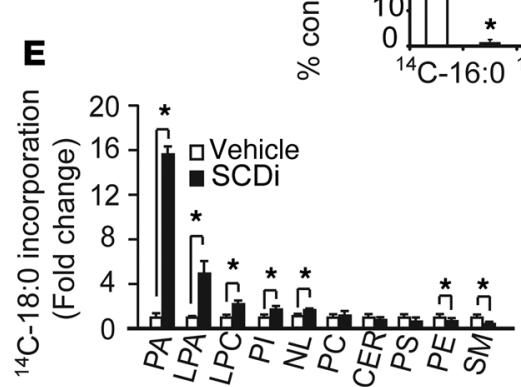

G

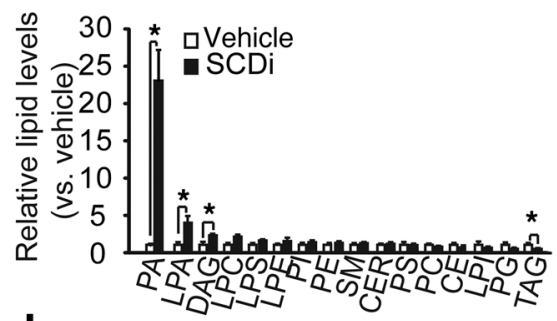

I

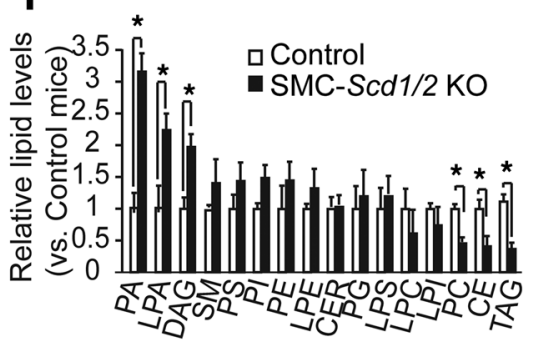

D

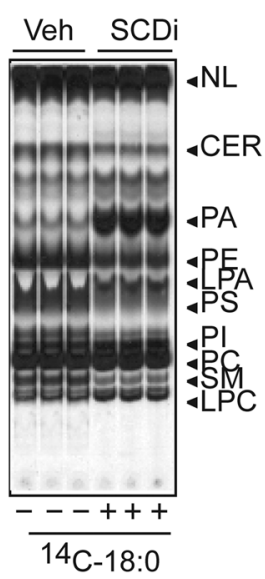

흐 14

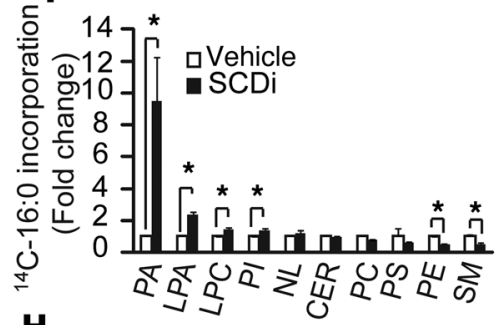

H

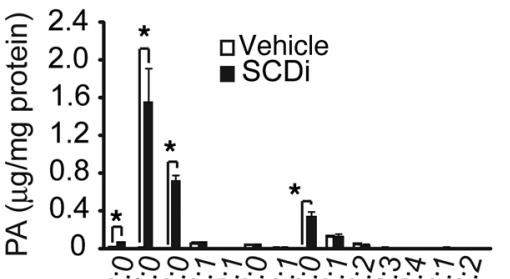

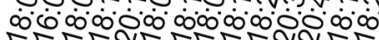

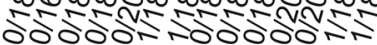

J

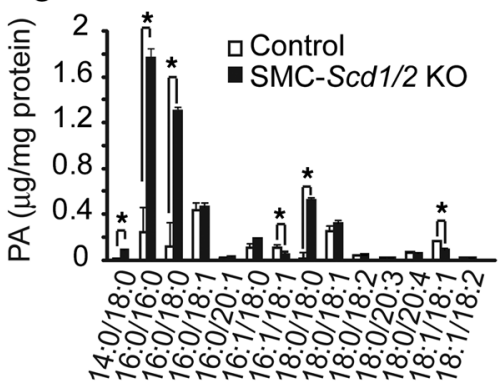

Figure 5. SFAs were preferentially incorporated into PA and accumulated as fully saturated PAs in VSMCs in vitro and in vivo. (A) Schematic representation of GPAT4, ACPAT3, and ACPAT5, which are localized in the ER. (B) Autoradiography and (C) quantification of SCD activity in human VSMCs treated with $300 \mathrm{nM}$ of the SCDi CAY10566 using ${ }^{14} \mathrm{C}-18: 0$ and ${ }^{14} \mathrm{C}-16: 0$ as substrates $(n=6)$. VSMCs were treated with ${ }^{14} \mathrm{C}-18: 0$ and ${ }^{14} \mathrm{C}-16: 0$ for 6 hours in the presence/absence of SCDi. SFAs and MUFAs isolated from total cell lysate were separated on a silver nitrate-coated TLC. (D) Autoradiography and quantification of (E) ${ }^{14} \mathrm{C}-18: 0$ and $(\mathbf{F})$ 16:0 incorporation into the lipid fraction in human VSMCs treated with SCDi. Human VSMCs were pretreated with SCDi for 2 hours and incubated with ${ }^{14} \mathrm{C}-18: 0$ and ${ }^{14} \mathrm{C}-16: 0$ for 6 hours in the presence/absence of SCDi. Total lipids isolated from total cell lysate (3 $\mathrm{mg}$ protein) were separated on a boric acid-coated TLC. (G) LC-MS-based lipidomic analysis and $(\mathbf{H})$ absolute levels of PA species in VSMCs treated with SCDi. Human VSMCs were treated with SCDi for 12 hours. Lipid content was quantified with LC-MS/MS. (I) LC-MS-based lipidomic analysis and (J) absolute levels of PA species in the aortic medial layers of SMC-Scd1/2 KO mice. Mice $(n=6)$ were sacrificed at 18 weeks old. The medial layer of aortas were dissected under a dissecting microscope. LPE, lysophosphatidylethanolamine; NL, neutral lipids. ${ }^{*} P<0.01$ (2-tailed Student's $t$ test).

UFAs such as 18:1n-9 reduce fully saturated PAs, resulting in a drastic attenuation of ER stress and vascular calcification. Cosupplementation with UFAs such as 18:1n-9 has been shown to rescue SFA-induced lipotoxicity through an unknown mechanism (25). Supplementation with exogenous UFAs such as 18:1n-9, linoleic acid (18:2) and docosahexaenoic acid (22:6) completely blocked SCD inhi-

and fully unsaturated PAs were not changed (Figure 5J). Similar to SMC-Scd1/2 KO mice, CKD DBA/2J mice had significantly higher levels of fully saturated PAs, such as 16:0/16:0-PA, 16:0/18:0-PA, and 18:0/18:0-PA (Supplemental Figure 9C), compared with sham-operated DBA/2J mice.

We previously found that 18:0 preferentially accumulates in the ER, which is highly associated with ER stress (5). We therefore hypothesized that SFAs, such as 18:0, accumulate in the form of PAs in the ER. To determine our hypothesis, we isolated the ER fraction from VSMCs treated with SCDi and analyzed levels of PA. LC-MS/MS analysis showed that fully saturated PAs such as 16:0/16:0-PA and 18:0/18:0-PA accumulate in the ER. Levels of fully saturated PAs in the ER fraction were $>10$-fold higher than in the whole cell lysates (Supplemental Figure 9D). bition-induced mineralization (Figure 6, A and B) and osteogenic differentiation (Figure 6C). In addition to SFA-induced mineralization, calcitriol-induced mineralization was inhibited by $18: 1 \mathrm{n}-9$ supplementation (Figure 6, D and E). UFA treatment completely blocked ER stress induced by SCD inhibition (Figure 6, F-H). We next examined whether UFAs modulate the lipid partitioning of SFAs. Borate-coated TLC analysis showed that UFA cotreatment completely rescued the accumulation of ${ }^{14} \mathrm{C}-18: 0$ into the PA fraction by SCD inhibition (Figure 6, I and J). In addition, quantitative LC-MS analysis showed that supplementation with 18:1n-9 completelynormalized levels of fully saturated PAs such as18:0/18:0-PA and 16:0/16:0-PA increased by SCD inhibition (Figure 6K).

Fully saturated PAs are disfavor substrates for LIPIN2, which contributes to its accumulation in SCD1/2-inhibited VSMCs. We 
A

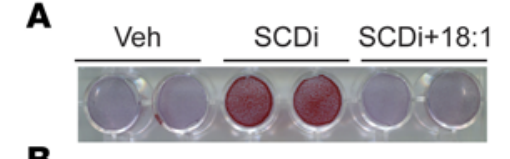

C
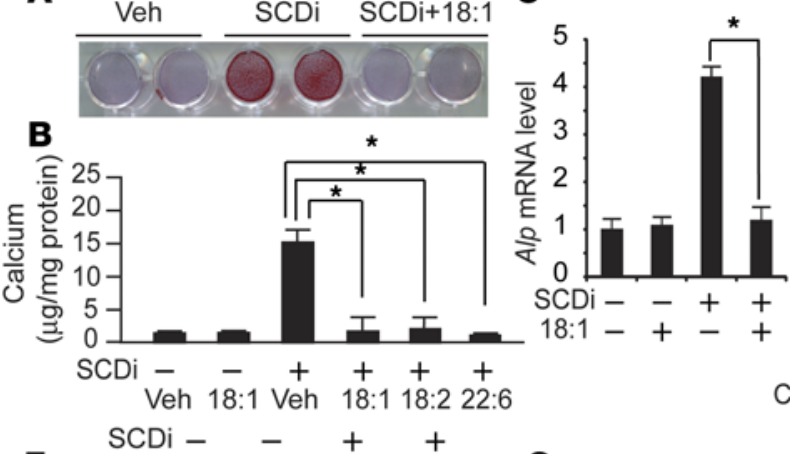

D
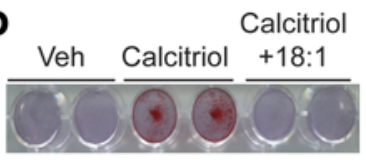

E

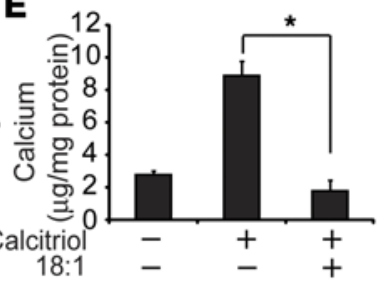

$\mathbf{F}$
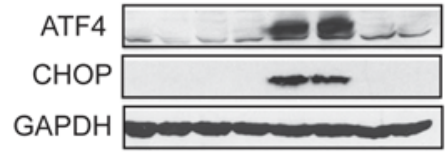

I

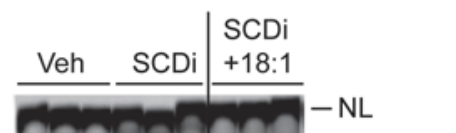

G

H

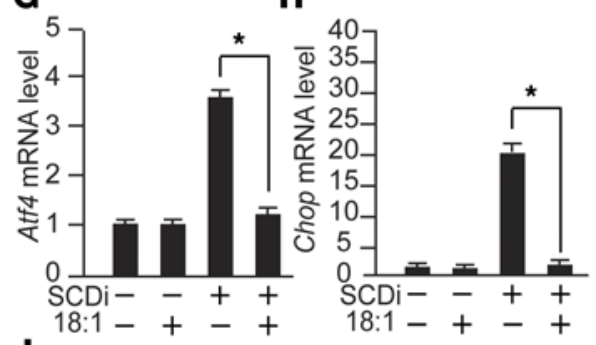

J

\section{$\mathbf{K}$}

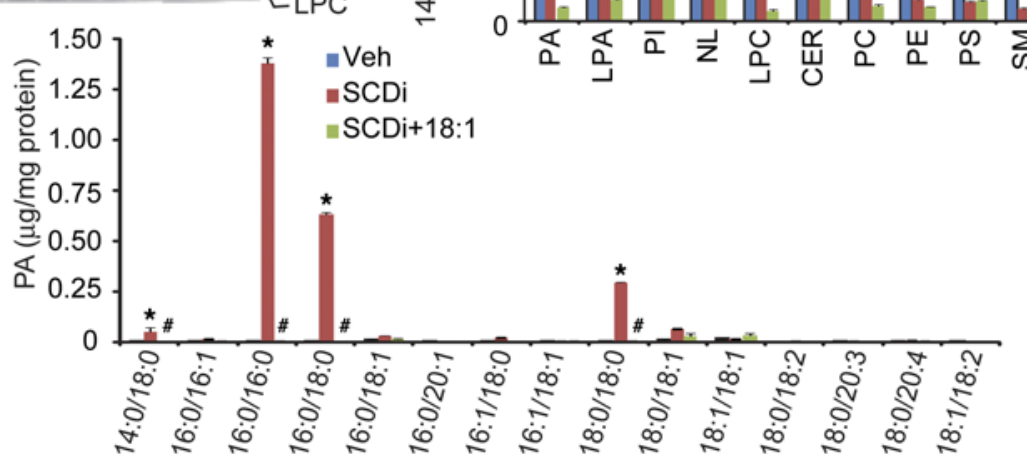

Figure 6. UFA cotreatment normalizes mineralization, osteogenic differentiation, ER stress, and PA accumulation. (A-C) UFAs blocked SCDi-induced mineralization (A and $\mathbf{B}$ ) and osteogenic differentiation (C) of VSMCs. Human VSMCs were treated with 200 $\mu \mathrm{M}$ UFAs in the presence of $300 \mathrm{nM}$ SCDi for 7 days in the presence of $2.0 \mathrm{mM}$ inorganic phosphate. (A) Seven days after the treatments, the cells were stained with Alizarin red to identify calcium deposits. (B) Calcium content was analyzed using a colorimetric assay. (C) Alp mRNA levels were analyzed by qPCR. (D and E) Calcitriol-induced mineralization is blocked by $18: 1 n-9$ supplementation. Human VSMCs were treated with $100 \mathrm{nM}$ calcitriol in the presence of $200 \mu \mathrm{M} 18: 1 n-9$ for 7 days. (F-H) UFAs such as 18:1n-9 completely blocked ER stress induced by SCDi. VSMCs were treated with SCDi acid for 24 hours. Total protein extracts were subjected to immunoblot analysis with ATF4- and CHOP-specific antibodies. Atf4 and Chop mRNA levels were quantified by qPCR. (I) Autoradiography and (J) quantification of ${ }^{14} \mathrm{C}-18: 0$ incorporation into the lipid fraction. Human VSMCs were treated with $200 \mu \mathrm{M} 18: 1 n-9$ in the presence of 300 $\mathrm{nM}$ SCDi and ${ }^{14} \mathrm{C}-18: 0(1 \mu \mathrm{Ci})$. The black line in I indicates that the image was derived from noncontiguous lanes of the same plate. Lipids were separated on a boric acid-coated TLC. (K) Absolute levels of PA species in VSMCs cotreated with SCDi and 18:1n-9. Human VSMCs were treated with $200 \mu \mathrm{M} 18: 1 n-9$ in the presence of $300 \mathrm{nM}$ SCDi for 24 hours. Each PA content was analyzed with LC-MS/ MS. NL, neutral lipids. $n=4,{ }^{*} P<0.01$ vs. vehicle (Veh) and ${ }^{\#} P<0.01$ vs. SCDi $(n=3-6$, one-way ANOVA). determined a mechanism by which SCD inhibition causes massive accumulation of fully saturated PAs in VSMCs, and UFA supplementation rescues the accumulation of fully saturated PAs. SCD inhibition and 18:0 treatment did not affect the expression and the activity of GPAT, AGPAT, and Lipin in VSMCs (data not shown). It has already been reported that GPAT4, AGPAT3, and AGPAT5 comparably utilize both saturated fatty acyl-CoAs such as 18:0-CoA and unsaturated acyl-CoAs such as 18:1-CoA. AGPAT3 and AGPAT5 also use both saturated and unsaturated LPA. We therefore hypothesized that LIPIN2 uses unsaturated PAs more efficiently than fully saturated PAs, which results in the specific accumulation of fully saturated PAs such as 18:0/18:0-PA and 16:0/16:0-PA in Scd1/2 knockdown VSMCs. To determine the substrate preference of LIPIN2, the lysate of HEK-293 cells infected with adenoviruses expressing human LIPIN2 was used as a recombinant LIPIN2 protein, whose activity was completely inhibited by N-methylmaleimide. The conversions of PAs to DAGs by the recombinant LIPIN2 were detected by LC-MS/MS. We used 16:0/16:0-PA and 18:0/18:0-PA as fully saturated PAs, 16:0/18:1-PA and 18:0/18:1-PA as partially unsaturated PAs, and 18:1/18:1-PA and 18:2/18:2-PA as fully unsaturated PAs. As shown in Figure 7A, LIPIN2 showed preferential specificity for fully unsaturated PAs (18:1/18:1-PA and 18:2/18:2-PA), followed next by partially unsaturated PAs (18:0/18:1-PA and 16:0/18:1-PA). Fully saturated PAs, 16:0/16:0-PA and 18:0/18:0-PA, were disfavor substrates for LIPIN2 and displayed only $1 \%$ of Lipin2 activity when compared with 18:1/18:1-PA (Figure 7A).

Fully saturated PAs such as 18:0/18:0-PA, but not unsaturated $P A s$ or partially unsaturated PAs, induced vascular calcification and lipotoxicity. To obtain direct evidence that fully saturated PAs 


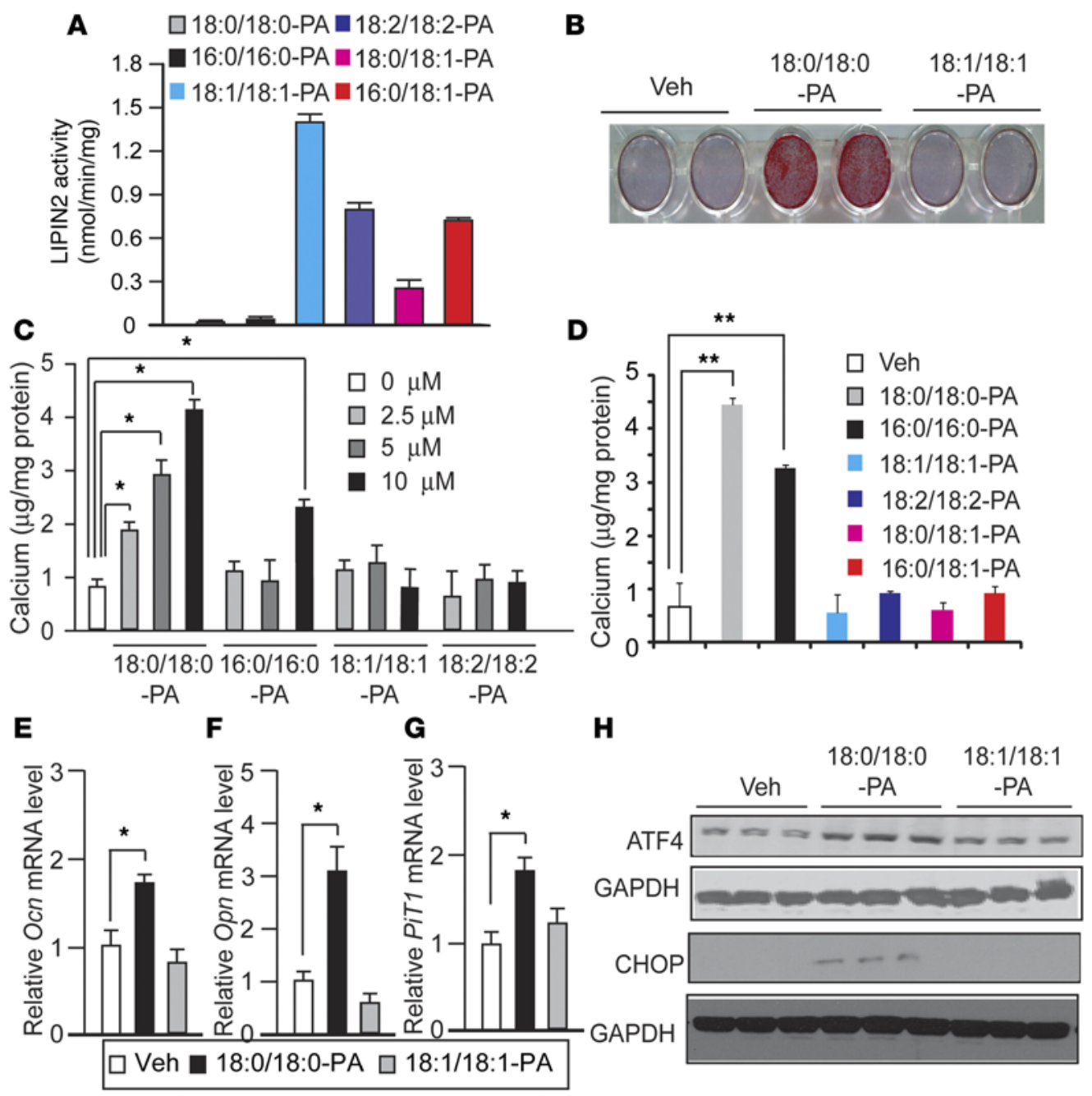

I

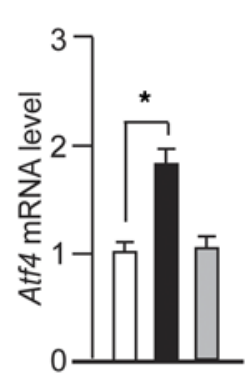

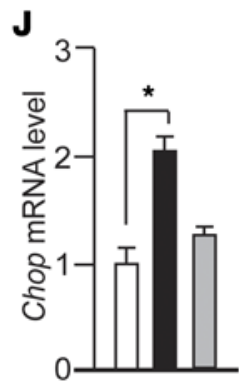

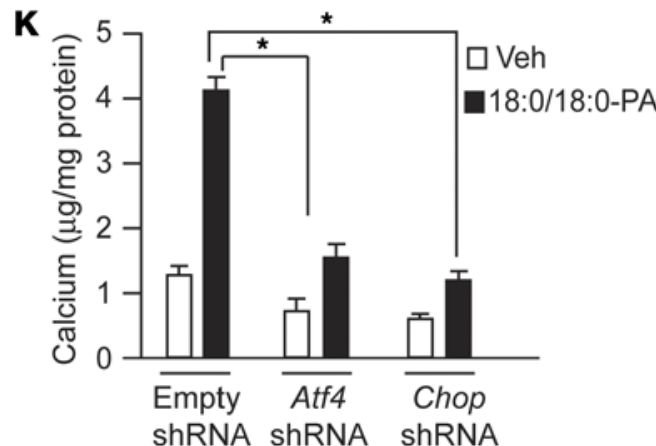

Figure 7. Fully unsaturated, but not partially saturated or fully saturated PAs, induce mineralization, osteogenic differentiation, and ER stress. (A)

Fully saturated PAs are disfavor substrates for LIPIN2. The lysate of HEK293 cells infected with adenovirus containing human LIPIN2 was used as a LIPIN2 recombinant protein. LIPIN2 activity was measured as the conversion of PA to DAC using LC-MS/MS. The fully saturated PAs 18:0/18:0-PA and 16:0/16:0-PA)but not the partially saturated PAs 18:0/18:1-PA and 16:0/18:1-PA or the fully unsaturated PAs 18:1/18:1-PA and 18:2/18:2-PA dose-dependently induced (B-D) mineralization and $(\mathbf{E}-\mathbf{C})$ osteogenic differentiation of VSMCs. VSMCs were treated with each PA for 7 days in the presence of $2.0 \mathrm{mM}$ phosphate. (B) Seven days after the treatments, the cells treated with PAs $(20 \mu \mathrm{M})$ were stained with Alizarin red to identify calcium deposits. (C and D) Calcium was extracted with $0.6 \mathrm{~N}$ hydrochloric acid and analyzed using a colorimetric assay. (E-C) Ocn, Opn, and PiT1 were quantified by qPCR. (H-J) Fully saturated PAs such as 18:0/18:0-PA, but not fully unsaturated PAs such as 18:1/18:1-PA, induce ER stress. VSMCs were treated with $10 \mu \mathrm{M}$ PAs for 6 and 24 hours. Total protein extracts were subjected to immunoblot analysis with ATF4and CHOP-specific antibodies. Atf4 and Chop mRNA levels were quantified by qPCR. (K) 18:0/18:0PA induces mineralization of VSMCs through the activation of the ATF4-CHOP axis of the ER stress response. Atf4 and Chop knockdown MOVAS cells were treated with $10 \mu \mathrm{M}$ 18:0/18:0-PA for 7 days. $n=6$; ${ }^{*} P<0.05$ (oneway ANOVA). induce vascular calcification and lipotoxicity, human VSMCs were treated with 18:0/18:0-PA and 16:0/16:0-PA as fully saturated PAs, and 18:1/18:1-PA and dilinoleyl-PA (18:2/18:2-PA) as a fully unsaturated PA. These PAs showed distinct effects on mineralization, ostogenic differentiation, ER stress, and lipotoxicity in VSMCs. As shown in Figure 7, B and C, the fully saturated PAs 18:0/18:0-PA and 16:0/16:0-PA significantly increased calcium content. In particular, 18:0/18:0-PA was very potent at inducing mineralization (Figure 7B). It increased calcium content as low as $2.5 \mu \mathrm{M}$ in a dose-dependent manner (Figure $7 \mathrm{C}$ ). At a $10 \mu \mathrm{M}$ concentration, 18:0/18:0-PA increased calcium content by over 5 -fold compared with the vehicle. The fully unsaturated PAs 18:1/18:1-PA and 18:2/18:2-PA and the partially unsaturated PAs 18:0/18:1-PA and 16:0/18:1 had no effect on mineralization (Figure 7D). Consistently, 18:0/18:0-PA but not 18:1/18:1-PA induced mRNA and protein expressions of osteogenic markers such as Ocn, Opn, and PiT1 (Figure 7, E-G), and ER stress makers such as ATF4 and CHOP (Figure 7, H-J). Cosupplementation with 18:1/18:1-PA did not affect 18:0/18:0-PA-induced mineralization (data not shown). The data suggest that the inhibitory effect of UFAs on SFA-induced mineralization requires the competition of free UFAs with free SFAs on the acylation to prevent the formation of fully saturated PAs. To 


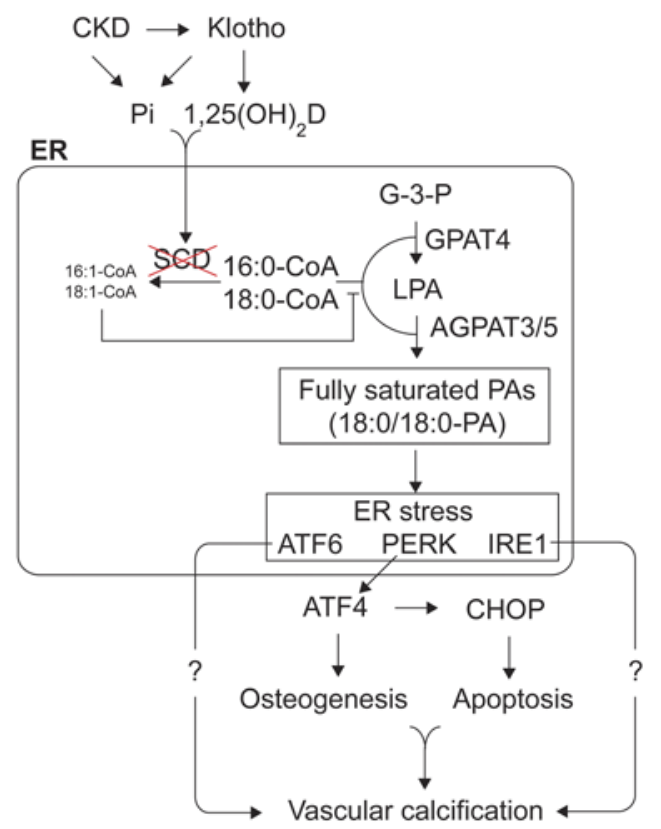

Figure 8. Schematic diagram of SFA-dependent vascular calcification. Increased SFAs through SCD inhibition leads to the accumulation of fully saturated PAs in the ER via de novo glycerolipid synthesis. The accumulation of fully saturated PAs induces ER stress responses such as ATF4-mediated osteogenesis and CHOP-mediated apoptosis, leading to vascular calcification. UFAs, however, competitively inhibit the formation of fully saturated PAs on the reaction of GPAT4, AGPAT3, and AGPAT5. Pi; inorganic phosphate; $1,2(\mathrm{OH})_{2} \mathrm{D}$, calcitriol; G-3-P, Glycerol-3-phosphate.

determine whether 18:0/18:0-PA induces mineralization through the ATF4-CHOP axis of the ER stress response, we treated Atf4 knockdown and Chop knockdown VSMCs with 18:0/18:0-PA. Atf4 and Chop knockdown significantly attenuated mineralization of VSMCs induced by 18:0/18:0-PA (Figure 7K).

\section{Discussion}

Vascular calcification is a common complication in patients who are aging, have diabetes, or have chronic kidney disease (44-46). However, there is no effective therapy currently available. We and other groups recently demonstrated that the accumulation of SFAs through either SCD inhibition or exogenous supplementation potently induces mineralization and osteogenic differentiation of VSMCs $(15,47)$. However, whether SCD inhibition and SFA accumulation causes vascular calcification in vivo was not determined. In this study, we demonstrated that the drastic repression of SCD expression in VSMCs is a characteristic event in animal models of vascular calcification. Klotho deficiency and CKD, which are major causative contributors to vascular calcification, significantly reduced SCD1 and SCD2 expression and its activity in the VSMCs of mice. In addition, levels of 18:0 and a significantly lower desaturation index (an in vivo marker for SCD activity) were also found in human patients with stages 3 and 4 CKD. Hyperphosphatemia and hypervitaminosis D are major contributors in klotho deficiency- and CKD-induced vascular calcification. We found that high-phosphate and calcitriol levels strongly reduced SCD expression and activity in human VSMCs. Thus, we concluded that the accumulation of $18: 0$ by a significant reduction of SCD by phosphate and calcitriol is a major event in the development of vascular calcification.

In addition, we demonstrated that dual inhibition of $s c d 1$ and $S c d 2$ directly causes vascular calcification, along with massive accumulation of SFAs both in vitro and in vivo. This is direct evidence that SFA-induced lipotoxicity through $S c d 1$ and $S c d 2$ repression in VSMCs plays a causative role in the pathogenesis of vascular calcification. There are several mechanisms by which SFA accumulation induces vascular calcification. Recent studies suggest that ER stress-mediated cell death contributes to the pathogenesis of vascular calcification (9). Atf4 and Chop knockdown attenuates mineralization and osteogenic differentiation of VSMCs in response to SFA treatment and other inductive factors $(5,6,9)$. In addition to saturated fat, oxidized lipids and TNF $\alpha$ - which induce ER stress, tunicamycin, and thapsigargin - also induce mineralization of VSMCs. We previously reported that in vivo CHOP deficiency and treatment with chemical chaperones such as 4-phenylbutyric acid and tauroursodeoxycholic acid attenuate CKD-dependent vascular calcification and inhibit cell apoptosis $(6,9)$. This study provides direct evidence that accumulation of SFAs by SCD inhibition induces vascular CHOP expression upon ER stress response activation, which in turn causes vascular apoptosis, thereby inducing vascular calcification. Our current study provides in vivo evidence that the accumulation of SFAs in VSMCs through SMC-specific SCD deficiency induces ATF4-mediated osteogenesis $(5,48)$ and CHOP-mediated cell death $(9,49)$, which synergistically lead to ectopic mineralization and osteogenic differentiation of VSMCs. We also found that, in addition to the PERK arms, the ATF6 and IRE1 arms of the ER stress response contribute to SFA-induced mineralization via unknown mechanisms. Identification of specific mechanisms by which the ATF6 and IRE1 pathways induce vascular calcification will be required in future studies.

In addition to vascular calcification, the ectopic accumulation of SFAs and their lipotoxic effects, including ER stress, oxidative stress, and apoptotic cell death, are implicated in the pathogenesis of other metabolic diseases such as diabetes, obesity, and atherosclerosis $(20,21,29,50,51)$. However, the molecular mechanism by which an SFA metabolite elicits these lipotoxic effects has not been identified. Our current findings are the first to demonstrate that fully saturated PAs generated though de novo glycerolipid synthesis are the major metabolites responsible for SFA-induced lipotoxicity in VSMCs. The first step to identifying the toxic metabolite was screening inhibitors and the shRNA library to identify 18:0-metabolizing enzymes responsible for lipotoxicity to consolidate potential SFA metabolites. The metabolism of 18:0 mainly includes fatty acid oxidation, desaturation, and acylation. We previously reported that (i) 18:0 most potently induced ER stress in VSMCs, (ii) acyl-CoA formation was required for 18:0-induced ER stress, and (iii) the conversion of 18:0 to 18:1n-9 by SCD was protective for 18:0-induced ER stress (5). Etomoxir, a fatty acid oxidation inhibitor, did not affect SFA-induced lipotoxicity, suggesting that fatty acid oxidation is a minor contributor in SFA-induced lipotoxicity. In addition, over $70 \%$ of $18: 0-\mathrm{CoA}$ is incorporated into lipid fractions by acyltransferases. We therefore hypothesized that a metabolite derived from SFAs through acylation mediates 
the lipotoxicity. Twenty acyltransferases were present in VSMCs. Based on the shRNA screening, we found that, out of these 21 acyltransferases expressed in VSMCs, GPAT4, AGPAT3, and AGPAT5 contribute to SFA-induced mineralization and ER stress. GPAT and AGPAT constitute the first 2 reactions of de novo biosynthesis of glycerolipids and generate PAs by utilizing saturated acyl-CoA, including 18:0-CoA, as a substrate. We next examined whether PA or its downstream metabolite is responsible for SFA-induced lipotoxicity in VSMCs. LIPIN, a PA phosphatase, catalyzes the third step of de novo glycerolipid synthesis by converting PA to DAG. The shRNA-mediated knockdown of Lipin2, which is the major isoform in VSMCs, augmented SFA-induced lipotoxicities, such as mineralization and ER stress, in contrast to Gpat4, Agpat3, and Agpat5 knockdowns. These results suggest that PA is the major metabolite responsible for SFA-induced lipotoxicity. In addition to the evidence from the shRNA library screening, radioactive SFA distribution and lipidomic techniques reveal that in vitro and in vivo SCD inhibition leads to the preferential accumulation of SFAs into the PA fraction. In addition, the fully saturated PA 18:0/18:0-PA but not the unsaturated PA 18:1/18:1-PA induces ER stress and inflammatory cytokine expression, resulting in mineralization and osteogenic differentiation of VSMCs. Although the molecular mechanism by which fully saturated PAs induce ER stress needs to be determined in a future study, we demonstrated that the ATF4CHOP axis of the ER stress response contributes to mineralization induced by fully saturated PAs.

We also demonstrated the mechanism for the accumulation of SFAs into the PA fraction and the mechanism by which UFAs block SFA-induced lipotoxicity. LIPIN2 is a major enzyme that metabolizes PA into DAG in VSMCs. LIPIN2 shows a preferential response to fully unsaturated PAs such as 18:1/18:1-PA and 18:2/18:2-PA compared with fully saturated PAs such as 18:0/18:0-PA and 16:0/16:0-PA. This preference causes accumulation of fully saturated PAs in SCD-inhibited VSMCs. Even when excess UFAs such as 18:1n-9 and 18:2 are treated with VSMCs, they never accumulate as PAs. Fully saturated PAs are therefore metabolized less than unsaturated PAs. UFAs elicit distinct roles in the context of lipotoxicity $(22,25)$. Cosupplementation with UFAs such as 18:1n-9 and 22:6 acid has been known to block SFA-induced lipotoxicity $(25,29)$. However, the mechanism by which UFAs attenuate SFA-induced lipotoxicity is poorly understood. Cotreatment with UFAs such as 18:1n-9 and 22:6 acid completely blocks mineralization, osteogenic differentiation, and ER stress of VSMCs induced by SCDi and calcitriol. We also find that UFA cotreatment completely normalizes the preferential distribution of SFAs to the PA fraction and absolute levels of fully saturated PAs in SCDinhibited VSMCs. The LIPIN2 substrate specificity accounts for the mechanism by which UFA cotreatment reduces levels of PA, resulting in the attenuation of SFA-induced lipotoxicity. Partially saturated PAs are better substrates for LIPIN2 than fully saturated PAs. In addition, partially saturated PAs such as 18:1/16:0-PA and 18:0/18:1-PA do not show lipotoxic effects in contrast to fully saturated PAs. These results demonstrate that the insertion of at least one molecule of UFA into PA increases Lipin2 activity, thereby preventing the accumulation of fully saturated PAs and their lipotoxicity. SCD inhibition also unexpectedly increases fully saturated DAGs, suggesting that other LIPIN isoforms and
PAP2 can efficiently utilize fully saturated PAs. In addition, we find that DGAT2 cannot utilize fully saturated DAGs as a substrate when saturated acyl-CoA is another substrate. The occurrence of lower substrate specificities of LIPIN2 and DGAT2 toward fully saturated PAs and DAGs, respectively, is consistent with Goldman and Vagelos's hypothesis that at least one UFA is required for triglyceride synthesis (52). This explains why SCD inhibition and excess SFA increase levels of PAs and DAGs, but also reduces levels of TAGs.

The current study suggests that the intracellular location of fully saturated PA synthesis and accumulation is important for SFA-induced lipotoxicity, since GPAT4 but not GPAT1 knockdown inhibits SFA-induced lipotoxicities such as ER stress and mineralization. Although both GPAT1 and GPAT4 catalyze the same reaction that converts glycerol-3-phosphate to 1-lysophosphatidic acid, GPAT1 and GPAT4 are localized in the mitochondria outer membrane and ER, respectively $(53,54)$. In addition, both isoforms are able to utilize saturated fatty acyl-CoAs, such as 18:0-CoA, as a substrate (55). The accumulation of fully saturated PAs in the ER fraction was more than 10-fold higher than in the whole cell fraction. AGPAT3, AGPAT5, and LIPIN2 are also located in the ER (56-58). These data suggest that the ER is a major organelle for synthesizing and accumulating fully saturated PAs, which directly lead to the lipotoxicity, particularly ER stress. However, it is still possible that GPAT1, rather than GPAT4, contributes to other SFA-induced lipotoxicities such as oxidative stress and mitochondrial dysfunction.

In addition to VSMCs, lipotoxic effects induced by SFAs have been observed in a variety of cell types, including cardiomyocytes, fibroblasts, pancreatic $\beta$-cells, hepatocytes, and macrophages (25, $28-30,51,59)$. The accumulation of fully saturated PAs by exogenous SFA addition or SCD inhibition is not limited to VSMCs. We detected that hepatocytes and 3T3 fibroblasts accumulate fully saturated PAs in response to SCD inhibition. Interestingly, recent studies have demonstrated that hepatic fully saturated PAs such as 16:0/16:0-PA, but not unsaturated PAs derived from de novo glycerolipid synthesis, inhibit the mTOR complex 2, leading to insulin resistance $(60,61)$. Therefore, fully saturated PAs may not only play a critical role in vascular calcification, but also in many other cardiometabolic diseases.

In conclusion, our findings reveal that increased fully saturated PAs through de novo glycerolipid synthesis promotes mineralization of VSMCs under procalcific conditions, including CKD. As indicated in Figure 8, we propose that CKD and klotho deficiency reduce SCD expression by increased inorganic phosphate and calcitriol. Reduced SCD activity leads to an increase in SFA levels in VSMCs. SFAs such as 18:0 and 16:0 preferentially accumulate in PA fractions via the reaction of GPAT4 and AGAPT3/5 in the ER. UFAs such as 18:1n-9 compete with SFAs on the reaction of GPAT4 and AGAPT3/5 to inhibit the formation of fully saturated PAs. The accumulated fully saturated PAs cause ER stress, resulting in the induction of the pro-osteogenic factor ATF4 and the proapoptotic factor CHOP. The pro-osteogenic and proapoptotic effects of the 2 ER stress effectors synergistically lead to vascular calcification. In addition to the PERK arm, the IRE1 and ATF6 arms of the ER stress response also contribute to SFA-induced mineralization via a currently unknown mechanism. 


\section{Methods}

Animals. C57Bl6 Scd1 conditional KO (lox/lox) mice (32), C57Bl6 klotho-deficient mice (39), and C57Bl6 SMMHC-Cre ${ }^{E R(T 2)}$ mice (62) were generated as previously described. C57Bl6 Rosa-tomatoTd reporter (63), C57Bl6 SMHHC-Cre/GFP mice (64), and DBA/2J mice were obtained from the Jackson Laboratory. Similar to $S c d 1^{l o x} /$ lox mice, $S c d 2^{l o x} / / o x$ mice were generated by floxing the exon 3 encoding the catalytic site of this enzyme (Supplemental Figure 10). In brief, DNA pools from a mouse strain 129/SV BAC library (ResGen Inc.) were screened by PCR using a primer pair that amplified the exon 4 portion of the $S c d 2$ gene. A genomic fragment was isolated from the Scd2-BAC clone and introduced into a plasmid that carries the MC1-TK selection cassette. A mini-targeting vector was constructed by cloning 4 contiguous PCR fragments: (i) a homology to the region immediately upstream of exon 3, (ii) a region that contains a loxP-flanked exon 3, (iii) an FRT-flanked $p G K$ promoter/EM7 promoter-NEO-pGHpA cassette, and (iii) a homology to the region immediately downstream of exon 3. The targeting vector was linearized and introduced by electroporation into murine SV/129 R1 embryonic stem cells. Karyotypically normal ES clones were microinjected into C57BL/6 blastocysts to produce chimeric founders at the University of Wisconsin-Madison Biotechnology Center's Transgenic Animal Facility. The $S c d 2^{l o x} /$ lox mice were crossed with Rosa-Flp mice and backcrossed 10 times with C57Bl6 mice. Scd $1^{\text {lox } / l o x}$ and $S c d 2^{l o x / l o x}$ mice were bred to generate $S c d 1 / 2^{l o x} / l o x$ mice. The resulting $S c d 1 / 2^{l o x} / l o x$ mice were intercrossed with C57Bl6 SMMHC$C r e^{E R(T 2)}$ mice to obtain SMMHC-Cre $e^{E R(T 2)} ; S c d 1^{l o x / l o x} ; S c d 2^{l o x} /$ lox mice. Since the SMMHC-Cre ${ }^{E R(T 2)}$ transgene was inserted on the Y chromosome, only males were used in this study. Five-week-old males were i.p. injected with either $1 \mathrm{mg}$ tamoxifen in vegetable oil or the vehicle for 5 consecutive days. The mice treated with tamoxifen and vehicle were used as SMC-specific $S c d 1 / 2 \mathrm{KO}$ mice and control mice, respectively. After the injections, the mice were maintained on a special diet (TD10364) for 10 weeks. For cell sorting, SMC$S c d 1 / 2$ KO mice were crossed with Rosa26-tomatoTd reporter mice.

Cell cultures. Human aortic VSMCs were obtained from Lonza. MOVAS-1 was provided by Mansoor Husain at the University of Toronto (Toronto, Canada) $(5,6)$. These VSMCs were cultured in DMEM containing 10\% FBS and $2 \mathrm{mM}$ inorganic phosphate in the absence/presence of $200 \mu \mathrm{M}$ 18:0-BSA complex, $300 \mathrm{nM}$ CAY10566 (Cayman Chemical), $100 \mathrm{nM}$ calcitriol (Cayman Chemical), $0.1 \mu \mathrm{g} / \mathrm{ml}$ tunicamycin (Enzo Life Science), $0.1 \mu \mathrm{M}$ thasigargin (Fisher Scientific), and various doses of PA (methyl$\beta$-cyclodextrin complex, Avanti Polar Lipids Inc.). Seven days after the treatments, cells were stained with Alizarin red to identify calcium deposits. Except for the experiments with shRNAs, human VSMCs were used in this study.

Generation of shRNA knockdown and VSMCs. MOVAS-1 cells were infected with recombinant lentiviruses containing gene-specific shRNAs (Open Biosystems). The shRNA clones are shown in Supplemental Table 2. Colonies were selected by treatment with $5 \mu \mathrm{g} / \mathrm{ml}$ puromycin for 7 days.

Histological analysis. Calcified lesions at the aortic valve were analyzed as previously described $(6,9,18,65)$. Apoptotic cells and CHOP in aortic sinuses were detected using an In Situ Cell Death Detection Kit (Roche Diagnostics) and a CHOP monoclonal antibody (Cell Signaling Technology), respectively, as previously described $(6,9,49)$.
Immunomagnetic cell sorting. Isolated aortas were digested to single cells by digestion at $37^{\circ} \mathrm{C}$ in collagenase buffer $(3.2 \mathrm{mg} / \mathrm{ml} \mathrm{col}-$ lagenase II, $0.7 \mathrm{mg} / \mathrm{ml}$ elastase, $0 / 2 \mathrm{mg} / \mathrm{ml}$ soybean trypsin inhibitor) in Hank's buffered saline solution. Aortas were harvested under sterile conditions following flushing of the vasculature system with sterile heparinized PBS, and minced prior to the digestion. Single cell suspension was sorted based on GFP and tomatoTd expression for klotho mice and SMC-Scd1/2 KO mice, respectively. Sorting was performed on a MoFlo high-speed cell sorter (Beckman Coutler) at the University of Colorado flow cytometry and sorting core facility.

Immunoblot analysis. Cell and tissue lysates were prepared using RIPA buffer (Cell Signaling Technoloy). The samples were separated by SDS-PAGE, transferred to a nitrocellulose membrane, and immunoblotted with the following antibodies: p-PERK (clone 16F8), p-eIF2 $\alpha$ (clone 119A11), CHOP (clone D46F1), and ATF4 (clone D4B8) from Cell Signaling Technology; CREB2 (clone C-20), GADD153 (clone B-3), SCD1 (clone S-15), SCD2 (clone C-16), and GAPDH (clone V-18) from Santa Cruz Biotechnology Inc.; GPAT4 (clone NB100-2389) and SCD5 (clone 45800002) from Novus Biologicals; and GPAT1 (clone 4613) from ProSci. Samples were visualized using horseradish peroxidase coupled to appropriate secondary antibodies, with enhancement by an ECL detection kit.

Calcium content in cultured cells and aortas. Calcium deposition in the plates and the aortas was quantified as previously described $(6,9,18)$.

RNA analysis. qPCR assays were performed using an Applied Biosystems StepOne qPCR instrument. Quantitative expression values were calculated from an absolute standard curve method using the plasmid template (Open Biosystems; GE Healthcare) containing each of the target gene cDNA. Primer sequences that are fully validated were obtained from the Primer Bank (http://pga.mgh.harvard. edu/primerbank; Harvard University, Cambridge, Massachusetts, USA) except for $s X B P-1$ and Ocn, as shown in Supplemental Table 5.

Generation of GPAT4- and LIPIN2-overexpression VSMCs using the Tet-on gene expression system. Human GPAT4 and human LIPIN2 cDNA were purchased from Open Biosystems and cloned into a pLenti-CMV-Zeo vector (Addgene). VSMCs expressing TetR (TetRVSMCs) were generated by infecting lentiviruses containing TetR and a single colony isolation with blasticidin $(5 \mu \mathrm{g} / \mathrm{ml})$. TetR-VSMCs were infected with lentiviruses containing either human GPAT4 or LIPIN2. Colonies were selected by treatment with $100 \mu \mathrm{g} / \mathrm{ml}$ zeocin for 7 days. To overexpress GPAT4 and LIPIN2, TetR-VSMCs expressing GPAT4 and LIPIN2 were treated with $1 \mathrm{mg} / \mathrm{ml}$ doxycycline (Dox) or vehicle for 24 hours and then treated with either $300 \mathrm{nM}$ CAY10566 or $200 \mu \mathrm{M}$ 18:0.

Determination of ${ }^{14} \mathrm{C}-18: 0,{ }^{14} \mathrm{C}-16: 0$, and ${ }^{14} \mathrm{C}-18: 1 n-9$ distribution. Control VSMCs were pretreated with either vehicle or $300 \mathrm{nM} \mathrm{SCDi.}$ After preincubation, VSMCs were treated with 1 or $5 \mu \mathrm{Ci}{ }^{14} \mathrm{C}$-steric acid or ${ }^{14} \mathrm{C}$-palmtic acid-BSA complex for 6 hours. Cells were harvested with PBS with buthylayted hydroxytoluene $(50 \mu \mathrm{g} / \mathrm{ml})$. Total lipids were extracted by Bligh \& Dyer method under acidic conditions. The extracted total lipids were subjected to $2.3 \%$ boric acidcoated TLC. The TLC was developed twice with chloroform/ethanol/ water/triethylamine (30:35:7:35), as previously described (66). The developed TLC was autoradiographed overnight. The resolving lipid fractions were scraped off the plate, and radioactivity was measured using a liquid scintillation counter. 
LC-MS lipidomic analysis. Lipid analysis was performed by LC electron spray ionization-MS/MS using a Simadzu LC-20AD LC system and an Applied Biosystems 3200 qTrap mass spectrometer with a turbo-iospray source $\left(350^{\circ} \mathrm{C}\right)$. For PA and phosphatidylglycerol (PG), LC was performed on a LC-NH2 $50 \times 2.1 \mathrm{~mm}$ column (Sigma-Aldrich). Solvents A and B consisted of acetonitrile/methanol/formic acid (97:2:1) containing $5 \mathrm{mM}$ ammonium formate and methanol/water/formic acid (89:6:5) containing $50 \mathrm{mM}$ triethylammonium acetate, respectively. Columns were heated to $50^{\circ} \mathrm{C}$. Lipids were separated under gradient conditions $(200 \mu \mathrm{l} / \mathrm{min})$ : $0 \%$ solvent $\mathrm{B}$ for 3 minutes, followed by a 1 minute linear gradient to $100 \%$ solvent $\mathrm{B}$, maintained for 3 minutes, restored to $100 \%$ Solvent $\mathrm{A}$ by a 1 minute linear gradient, maintained at $100 \%$ Solvent B for 5 minutes, returned to $0 \%$ solvent $\mathrm{B}$ at 11 minutes, and maintained for 5 minutes. Negative ion mode was used for PA and PG analysis. For other lipid analysis, LC was performed on a Zorbax C18, $1.8 \mu \mathrm{m}$, $50 \times 2.1 \mathrm{~mm}$ column (Agilent Technologies) as previously described. Solvents C and D consisted of tetrahydrofuran/methanol/water (30:20:50 and 75:20:5, respectively), both containing $10 \mathrm{mM}$ ammonium formate. Columns were heated to $50^{\circ} \mathrm{C}$. Lipids were separated under gradient conditions (300 $\mu \mathrm{l} / \mathrm{min})$ : $0 \%$ solvent D to $100 \%$ solvent $\mathrm{D}$ for 10.5 minutes, returned to $0 \%$ solvent $\mathrm{D}$ at 11 minutes, and maintained for 5 minutes. LPA, lysophosphatidylserine (LPS), lysophosphatidylinositol (LPI), PE, phosphatidylserine (PS), PG, and PI were analyzed with negative ion mode, whereas positive ion mode was used for phosphatidylcholine (PC), LPC ceramide (CER), DAG, TAG, CE, and SM. The detailed mass spectrometry conditions such as collision energy, declustering potential, and mass transition are shown in Supplemental Table 6. Internal standards were purchased from Avanti Polar Lipids.

Generation of adenoviruses. Adenoviruses containing human LIPIN2 cDNA, DGAT 2 cDNA, Scd1 shRNA, and GFP shRNA were generated using a ViraPower Adenoviral Expression System (Invitrogen).

SCD activity. SCD activity was measured as previously described $(31,32,67,68)$. For the microsomal SCD assay, microsomes were incubated with ${ }^{14} \mathrm{C}-18: 0-\mathrm{CoA}(1 \mu \mathrm{Ci})$ and NADH $(20 \mathrm{mM})$ for 5 minutes. For the cell assay, cells were incubated with ${ }^{14} \mathrm{C}-18: 0$ or ${ }^{14} \mathrm{C}-16: 0-\mathrm{BSA}$ complex for 16 hours. After the reaction, the extracted and saponified fatty acids were separated by $10 \%$ silver nitrate-impregnated TLC using chloroform/methanol/acetic acid/water (90:8:1:0.8) as the developing solution. The plates were autoradiographed. Fractions were scraped off of the plate, and radioactivity was measured using a liquid scintillation counter.

PAP and DGAT activity. PAP activity was measured on cell extracts, as described previously with a modification $(69,70)$. Cell extracts from HEK293AD cells treated with adenovirus containing human LIPIN2 were used as a crude LIPIN2 enzyme. Cells were harvested with a buffer containing $0.25 \mathrm{M}$ sucrose, $2 \mathrm{mM}$ dithiothreitol (to stabilize PAP1 activity), protease inhibitor mixture, phosphatase inhibitor cocktail, and $0.15 \%$ Tween 20. We designed an optimum assay for PAP using $100 \mathrm{mM}$ Tris/maleate buffer (pH 6.5), $5 \mathrm{mM}$ $\mathrm{MgCl} 2,2 \mathrm{mg} / \mathrm{ml}$ fatty acid-poor bovine serum albumin, and $0.6 \mathrm{mM}$ PA, which was dispersed in $0.4 \mathrm{mM}$ egg PC and $1 \mathrm{mM}$ EDTA that was used to prepare the lipid substrate. The conversion of PA to DAG by PAP-1 was analyzed by LC-MS. PAP activity in the LIPIN2 enzyme was completely inhibited in the presence of $5 \mathrm{mM}$ N-ethylmaleimide. The PAs 18:0/18:0, 16:0/16:0, 18:1/18:1, 16:0/18:1-PA, 18:0/18:1-PA, and 18:2/18:2 were purchased from Avanti Polar Lipids. DGAT activity was measured on microsomal fraction, as described previously, with a modification (71). Microsomal fraction from HEK293AD cells treated with adenovirus containing human DGAT2 was used as a crude DGAT2 enzyme. Crude DGAT2 $(1 \mu \mathrm{g})$ was incubated for $5 \mathrm{~min}$ utes at $37^{\circ} \mathrm{C}$ with $25 \mu \mathrm{M}$ 18:0-CoA and $200 \mu \mathrm{M}$ DAGs (18:0/18:0-DAG and 18:1/18:1-DAG) from Nu Check. The conversion of DAG to TAG by DAGT2 was analyzed by LC-MS. The conditions of LC-MS were described in the above section of the LC-MS-based lipidomic analysis.

Biochemical analysis. Serum cholesterol, triglyceride, phosphorus, and calcium were analyzed using colorimetric enzyme assay kits (Pointe Scientific). Serum FGF23 was measured using an FGF23 ELISA kit (Immutopics International).

Human subjects. Serum samples were collected from 10 patients with stage 3 and 4 CKD (mean eGFR $34 \pm 7 \mathrm{ml} / \mathrm{min} / 1.73 \mathrm{~m}^{2}$ ) and mean age (57 \pm 7 years), and 10 age-matched patients with normal kidney function (mean eGFR $80 \pm 12 \mathrm{ml} / \mathrm{min} / 1.73 \mathrm{~m}^{2}$ ) and mean age ( $59 \pm 14$ years) after a minimum 4 -hour fast. Trained personnel at the University of Colorado Clinical and Translational Research Center collected the serum samples. All patients gave informed consent for their blood specimen to be used for research purposes. Glomerular filtration rate (GFR) was estimated using the 4 -variable modification of diet in renal disease prediction equation. All other parameters, such as serum cholesterol, phosphorus, and calcium, were previously reported (9). The nature, benefits and risks of the study were explained to the volunteers, and their written informed consent was obtained prior to participation.

Statistics. Data were collected from more than 2 independent experiments and were reported as the mean \pm SEM. Statistical analysis for 2-group comparison was performed using a 2-tailed Student's $t$ test, or one-way ANOVA with a Student-Newman-Keuls post-hoc test for multigroup comparison. Significance was accepted at $P<0.05$.

Study approval. Animal experiments were approved by the Institutional Animal Care and Research Advisory Committee of the University of Colorado at Denver. All human participants were enrolled in a separate clinical research study, and the procedures were approved by the Colorado Multiple Institutional Review Board or the Institutional Review Board of the University of Colorado Boulder.

\section{Author contributions}

M. Masuda, SMA, and M. Miyazaki designed and performed the main experiments, and ALK and KO provided technical assistance for the main experiments. MK, JMN, and SO provided essential mouse lines for the completion of the studies and assisted with manuscript preparation. JK and MC provided human serum samples. M. Masuda, SMA, $\mathrm{ALK}, \mathrm{KO}$, and M. Miyazaki discussed and interpreted the results from the study. M. Miyazaki, ALK, and M. Masuda wrote the paper.

\section{Acknowledgments}

The authors work was supported by grants, including NIH DK096030 and HL117602 to M. Miyazaki and DK62388 to J.M. Ntambi. M. Masuda received a fellowship from the American Heart Association (13POST13820008).

Address correspondence to: Makoto Miyazaki, 12700 E. 17th Ave., C281 RC-2, Rm 7450, Aurora, Colorado 80045, USA. Phone: 303.724.4828; E-mail: makoto.miyazaki@ucdenver.edu. 
1. Towler DA. Molecular and cellular aspects of calcific aortic valve disease. Circ Res. 2013;113(2):198-208.

2. Demer LL, Tintut Y. Inflammatory, metabolic, and genetic mechanisms of vascular calcification. Arterioscler Thromb Vasc Biol. 2014;34(4):715-723.

3. Cheng SL, et al. Targeted reduction of vascular Msx1 and Msx2 mitigates arteriosclerotic calcification and aortic stiffness in LDLRdeficient mice fed diabetogenic diets. Diabetes. 2014;63(12):4326-4337.

4. Sun Y, et al. Smooth muscle cell-specific runx2 deficiency inhibits vascular calcification. Circ Res. 2012;111(5):543-552.

5. Masuda M, Ting TC, Levi M, Saunders SJ, Miyazaki-Anzai S, Miyazaki M. Activating transcription factor 4 regulates stearate-induced vascular calcification. J Lipid Res. 2012;53(8):1543-1552.

6. Masuda M, Miyazaki-Anzai S, Levi M, Ting TC, Miyazaki M. PERK-eIF2alpha-ATF4-CHOP signaling contributes to TNF $\alpha$-induced vascular calcification. J Am Heart Assoc. 2013;2(5):e000238.

7. Geng Y, et al. The role of cellular cholesterol metabolism in vascular cell calcification. J Biol Chem. 2011;286(38):33701-33706.

8. Baigent $\mathrm{C}$, et al. The effects of lowering LDL cholesterol with simvastatin plus ezetimibe in patients with chronic kidney disease (Study of Heart and Renal Protection): a randomised placebo-controlled trial. Lancet. 2011;377(9784):2181-2192.

9. Miyazaki-Anzai S, et al. Endoplasmic reticulum stress effector CCAAT/enhancer-binding protein homologous protein (CHOP) regulates chronic kidney disease-induced vascular calcification. JAm Heart Assoc. 2014;3(3):e000949.

10. Mody N, Parhami F, Sarafian TA, Demer LL. Oxidative stress modulates osteoblastic differentiation of vascular and bone cells. Free Radic Biol Med. 2001;31(4):509-519.

11. Crouthamel MH, et al. Sodium-dependent phosphate cotransporters and phosphate-induced calcification of vascular smooth muscle cells: redundant roles for PiT-1 and PiT-2. Arterioscler Thromb Vasc Biol. 2013;33(11):2625-2632.

12. El-Abbadi MM, et al. Phosphate feeding induces arterial medial calcification in uremic mice: role of serum phosphorus, fibroblast growth factor-23, and osteopontin. Kidney Int. 2009;75(12):1297-1307.

13. Tintut Y, Patel J, Parhami F, Demer LL. Tumor necrosis factor-alpha promotes in vitro calcification of vascular cells via the cAMP pathway. Circulation. 2000;102(21):2636-2642.

14. Abedin M, Lim J, Tang TB, Park D, Demer LL, Tintut $\mathrm{Y}$. N-3 fatty acids inhibit vascular calcification via the p38-mitogen-activated protein kinase and peroxisome proliferator-activated receptor- $\gamma$ pathways. Circ Res. 2006;98(6):727-729.

15. Kageyama A, et al. Palmitic acid induces osteoblastic differentiation in vascular smooth muscle cells through ACSL3 and NF- $\mathrm{B}$, novel targets of eicosapentaenoic acid. PLoS One. 2013;8(6):e68197.

16. Watson KE, Bostrom K, Ravindranath R, Lam T, Norton B, Demer LL. TGF- $\beta 1$ and 25-hydroxycholesterol stimulate osteoblast-like vascular cells to calcify. J Clin Invest. 1994;93(5):2106-2113.

17. Parhami F, et al. Lipid oxidation products have opposite effects on calcifying vascular cell and bone cell differentiation. Arterioscler Thromb Vasc Biol. 1997;17(4):680-687.

18. Miyazaki-Anzai S, Levi M, Kratzer A, Ting TC, Lewis LB, Miyazaki M. FXR activation prevents the development of vascular calcification in $\mathrm{ApoE}^{-/-}$mice with chronic kidney disease. Circ Res. 2010;106(12):1807-1817.

19. Ting TC, et al. Increased lipogenesis and stearate accelerate vascular calcification in calcifying vascular cells. J Biol Chem. 2011;286(27):23938-23948.

20. Unger RH. Lipotoxicity in the pathogenesis of obesity-dependent NIDDM. Diabetes. 1995;44(8):863-870.

21. Unger RH, Orci L. Lipoapoptosis: its mechanism and its diseases. Biochim Biophys Acta. 2002;1585(2-3):202-212.

22. Brookheart RT, Michel CI, Schaffer JE. As a matter of fat. Cell Metab. 2009;10(1):9-12.

23. Michel CI, et al. Small nucleolar RNAs U32a, U33, and $\mathrm{U} 35 \mathrm{a}$ are critical mediators of metabolic stress. Cell Metab. 2011;14(1):33-44.

24. Brodeur MR, Bouvet C, Barrette M, Moreau P. Palmitic acid increases medial calcification by inducing oxidative stress. JVasc Res. 2013;50(5):430-441.

25. Listenberger LL, et al. Triglyceride accumulation protects against fatty acid-induced lipotoxicity. Proc Natl Acad Sci U S A. 2003;100(6):3077-3082.

26. Wei Y, Wang D, Gentile CL, Pagliassotti MJ. Reduced endoplasmic reticulum luminal calcium links saturated fatty acid-mediated endoplasmic reticulum stress and cell death in liver cells. Mol Cell Biochem. 2009;331(1-2):31-40.

27. Marsh JB, James AT. The conversion of stearic to oleic acid by liver and yeast preparations. Biochim Biophys Acta. 1962;60:320-328.

28. Borradaile NM, Han X, Harp JD, Gale SE, Ory DS, Schaffer JE. Disruption of endoplasmic reticulum structure and integrity in lipotoxic cell death. JLipid Res. 2006;47(12):2726-2737.

29. Wei Y, Wang D, Topczewski F, Pagliassotti MJ. Saturated fatty acids induce endoplasmic reticulum stress and apoptosis independently of ceramide in liver cells. Am J Physiol Endocrinol Metab. 2006;291(2):E275-E281.

30. Anderson EK, Hill AA, Hasty AH. Stearic acid accumulation in macrophages induces toll-like receptor $4 / 2$-independent inflammation leading to endoplasmic reticulum stressmediated apoptosis. Arterioscler Thromb Vasc Biol. 2012;32(7):1687-1695.

31. Cohen P, et al. Role for stearoyl-CoA desaturase-1 in leptin-mediated weight loss. Science. 2002;297(5579):240-243.

32. Miyazaki M, et al. Hepatic stearoyl-CoA desaturase-1 deficiency protects mice from carbohydrate-induced adiposity and hepatic steatosis. Cell Metab. 2007;6(6):484-496.

33. Miyazaki M, Ntambi JM. Role of stearoylcoenzyme A desaturase in lipid metabolism. Prostaglandins Leukot Essent Fatty Acids. 2003;68(2):113-121.

34. Ntambi JM, Miyazaki M. Recent insights into
stearoyl-CoA desaturase-1. Curr Opin Lipidol. 2003;14(3):255-261.

35. Ntambi JM, Miyazaki M, Dobrzyn A. Regulation of stearoyl-CoA desaturase expression. Lipids. 2004;39(11):1061-1065.

36. Ntambi JM, Miyazaki M. Regulation of stearoylCoA desaturases and role in metabolism. Prog Lipid Res. 2004;43(2):91-104.

37. Minville-Walz M, et al. Distinct regulation of stearoyl-CoA desaturase 1 gene expression by cis and trans C18:1 fatty acids in human aortic smooth muscle cells. Genes Nutr. 2012;7(2):209-216.

38. Hu MC, et al. Klotho deficiency causes vascular calcification in chronic kidney disease. J Am Soc Nephrol. 2011;22(1):124-136.

39. Kuro-o M, et al. Mutation of the mouse klotho gene leads to a syndrome resembling ageing. Nature. 1997;390(6655):45-51.

40. Memon F, El-Abbadi M, Nakatani T, Taguchi T, Lanske B, Razzaque MS. Does Fgf23-klotho activity influence vascular and soft tissue calcification through regulating mineral ion metabolism? Kidney Int. 2008;74(5):566-570.

41. Ohnishi M, Nakatani T, Lanske B, Razzaque MS. Reversal of mineral ion homeostasis and soft-tissue calcification of klotho knockout mice by deletion of vitamin D 1alpha-hydroxylase. Kidney Int. 2009;75(11):1166-1172.

42. Wang J, et al. Characterization of HSCD5, a novel human stearoyl-CoA desaturase unique to primates. Biochem Biophys Res Commun. 2005;332(3):735-742.

43. Fagone P, Jackowski S. Membrane phospholipid synthesis and endoplasmic reticulum function. J Lipid Res. 2009;50(suppl):S311-S316.

44. Sage AP, Tintut Y, Demer LL. Regulatory mechanisms in vascular calcification. Nat Rev Cardiol. 2010;7(9):528-536.

45. Shanahan CM, Crouthamel MH, Kapustin A, Giachelli CM. Arterial calcification in chronic kidney disease: key roles for calcium and phosphate. Circ Res. 2011;109(6):697-711.

46. Mizobuchi M, Towler D, Slatopolsky E. Vascular calcification: the killer of patients with chronic kidney disease. J Am Soc Nephrol. 2009;20(7):1453-1464

47. Masuda M, et al. Regulation of renal sodiumdependent phosphate co-transporter genes (Npt2a and Npt2c) by all-trans-retinoic acid and its receptors. Biochem J. 2010;429(3):583-592.

48. Yang X, et al. ATF4 is a substrate of RSK2 and an essential regulator of osteoblast biology; implication for Coffin-Lowry Syndrome. Cell. 2004;117(3):387-398.

49. Thorp E, Li G, Seimon TA, Kuriakose G, Ron D, Tabas I. Reduced apoptosis and plaque necrosis in advanced atherosclerotic lesions of Apoe ${ }^{-/-}$ and $\mathrm{Ldlr}^{-/}$mice lacking CHOP. Cell Metab 2009;9(5):474-481.

50. Erbay E, et al. Reducing endoplasmic reticulum stress through a macrophage lipid chaperone alleviates atherosclerosis. Nat Med. 2009;15(12):1383-1391.

51. Rong X, et al. LXRs regulate ER stress and inflammation through dynamic modulation of membrane phospholipid composition. Cell Metab. 2013;18(5):685-697. 
52. Goldman P, Vagelos PR. The specificity of triglyceride synthesis from diglycerides in chicken adipose tissue. J Biol Chem. 1961;236:2620-2623.

53. Wendel AA, Cooper DE, Ilkayeva OR, Muoio DM, Coleman RA. Glycerol-3-phosphate acyltransferase (GPAT)-1, but not GPAT4, incorporates newly synthesized fatty acids into triacylglycerol and diminishes fatty acid oxidation. J Biol Chem. 2013;288(38):27299-27306.

54. Wendel AA, Lewin TM, Coleman RA. Glycerol-3-phosphate acyltransferases: rate limiting enzymes of triacylglycerol biosynthesis. Biochim Biophys Acta. 2009;1791(6):501-506.

55. Chen YQ, et al. AGPAT6 is a novel microsomal glycerol-3-phosphate acyltransferase. J Biol Chem. 2008;283(15):10048-10057.

56. Prasad SS, Garg A, Agarwal AK. Enzymatic activities of the human AGPAT isoform 3 and isoform 5: localization of AGPAT5 to mitochondria. J Lipid Res. 2011;52(3):451-462.

57. Wilfling F, et al. Triacylglycerol synthesis enzymes mediate lipid droplet growth by relocalizing from the ER to lipid droplets. Dev Cell. 2013;24(4):384-399.

58. Zhang P, Verity MA, Reue K. Lipin-1 regulates autophagy clearance and intersects with statin drug effects in skeletal muscle. Cell Metab. 2014;20(2):267-279.

59. Maedler K, Spinas GA, Dyntar D, Moritz W,
Kaiser N, Donath MY. Distinct effects of saturated and monounsaturated fatty acids on beta-cell turnover and function. Diabetes. 2001;50(1):69-76.

60. Zhang C, Wendel AA, Keogh MR, Harris TE, Chen J, Coleman RA. Glycerolipid signals alter mTOR complex 2 (mTORC2) to diminish insulin signaling. Proc Natl Acad Sci U S A. 2012;109(5):1667-1672.

61. Zhang C, et al. Inhibited insulin signaling in mouse hepatocytes is associated with increased phosphatidic acid but not diacylglycerol. J Biol Chem. 2015;290(6):3519-3528.

62. Wirth A, et al. G12-G13-LARG-mediated signaling in vascular smooth muscle is required for salt-induced hypertension. Nat Med. 2008;14(1):64-68.

63. Madisen L, et al. A robust and high-throughput Cre reporting and characterization system for the whole mouse brain. Nat Neurosci. 2010;13(1):133-140.

64. Xin HB, Deng KY, Rishniw M, Ji G, Kotlikoff MI. Smooth muscle expression of Cre recombinase and eGFP in transgenic mice. Physiol Genomics. 2002;10(3):211-215.

65. Miyazaki-Anzai S, Masuda M, Levi M, Keenan AL, Miyazaki M. Dual activation of the bile acid nuclear receptor FXR and G-protein-coupled receptor TGR5 protects mice against atheroscle- rosis. PLoS One. 2014;9(9):e108270.

66. Weerheim AM, Kolb AM, Sturk A, Nieuwland R. Phospholipid composition of cell-derived microparticles determined by one-dimensional high-performance thin-layer chromatography. Anal Biochem. 2002;302(2):191-198.

67. Miyazaki M, Kim YC, Gray-Keller MP, Attie AD, Ntambi JM. The biosynthesis of hepatic cholesterol esters and triglycerides is impaired in mice with a disruption of the gene for stearoyl-CoA desaturase 1. JBiol Chem. 2000;275(39):30132-30138.

68. Miyazaki M, Kim HJ, Man WC, Ntambi JM. Oleoyl-CoA is the major de novo product of stearoyl-CoA desaturase 1 gene isoform and substrate for the biosynthesis of the Harderian gland 1-alkyl-2,3-diacylglycerol. J Biol Chem. 2001;276(42):39455-39461.

69. Donkor J, Sariahmetoglu M, Dewald J, Brindley DN, Reue K. Three mammalian lipins act as phosphatidate phosphatases with distinct tissue expression patterns. J Biol Chem. 2007;282(6):3450-3457.

70. Dwyer JR, et al. Mouse lipin-1 and lipin-2 cooperate to maintain glycerolipid homeostasis in liver and aging cerebellum. Proc Natl Acad Sci U S A. 2012;109(37):E2486-E2495.

71. Harris CA, et al. DGAT enzymes are required for triacylglycerol synthesis and lipid droplets in adipocytes. J Lipid Res. 2011;52(4):657-667. 